\title{
Genetic predisposition to ductal carcinoma in situ of the breast
}

Christos Petridis ${ }^{1,2}$, Mark N. Brook ${ }^{3}$, Vandna Shah ${ }^{1}$, Kelly Kohut ${ }^{4}$, Patricia Gorman ${ }^{4}$, Michele Caneppele ${ }^{4}$, Dina Levi ${ }^{1}$, Efterpi Papouli ${ }^{5}$, Nick Orr ${ }^{6}$, Angela Cox ${ }^{7}$, Simon S. Cross ${ }^{8}$, Isabel dos-Santos-Silva ${ }^{9}$, Julian Peto ${ }^{9}$, Anthony Swerdlow ${ }^{3,10}$, Minouk J. Schoemaker ${ }^{3}$, Manjeet K. Bolla ${ }^{11}$, Qin Wang ${ }^{11}$, Joe Dennis ${ }^{11}$, Kyriaki Michailidou ${ }^{11}$, Javier Benitez ${ }^{12,13}$, Anna González-Neira ${ }^{12}$, Daniel C. Tessier ${ }^{14}$, Daniel Vincent ${ }^{14}$, Jingmei Li ${ }^{15}$, Jonine Figueroa ${ }^{16}$, Vessela Kristensen ${ }^{17,18,19}$, Anne-Lise Borresen-Dale ${ }^{17,18}$, Penny Soucy ${ }^{20}$, Jacques Simard ${ }^{20}$, Roger L. Milne ${ }^{21,22}$, Graham G. Giles ${ }^{21,22}$, Sara Margolin ${ }^{23}$, Annika Lindblom ${ }^{24}$, Thomas Brüning ${ }^{25}$, Hiltrud Brauch ${ }^{26,27,28}$, Melissa C. Southey ${ }^{29}$, John L. Hopper ${ }^{22}$, Thilo Dörk ${ }^{30}$, Natalia V. Bogdanova ${ }^{31}$, Maria Kabisch ${ }^{32}$, Ute Hamann ${ }^{32}$, Rita K. Schmutzler ${ }^{33,34,35}$, Alfons Meindl ${ }^{36}$, Hermann Brenner $28,37,38$, Volker Arndt ${ }^{37}$, Robert Winqvist ${ }^{39,40}$, Katri Pylkäs ${ }^{39,40}$, Peter A. Fasching ${ }^{41,42}$, Matthias W. Beckmann ${ }^{41}$, Jan Lubinski ${ }^{43}$, Anna Jakubowska ${ }^{43}$, Anna Marie Mulligan ${ }^{44,45}$, Irene L. Andrulis ${ }^{46,47}$, Rob A. E. M. Tollenaar ${ }^{48}$, Peter Devilee ${ }^{49,50}$, Loic Le Marchand ${ }^{51}$, Christopher A. Haiman '52, Arto Mannermaa ${ }^{53,54,55}$, Veli-Matti Kosma ${ }^{53,54,55}$, Paolo Radice ${ }^{56}$, Paolo Peterlongo ${ }^{57}$, Frederik Marme ${ }^{58,59}$, Barbara Burwinkel ${ }^{59,60}$, Carolien H. M. van Deurzen ${ }^{61}$, Antoinette Hollestelle ${ }^{62}$, Nicola Miller ${ }^{63}$, Michael J. Kerin ${ }^{63}$, Diether Lambrechts ${ }^{64,65}$, Giuseppe Floris ${ }^{66}$, Jelle Wesseling ${ }^{67}$, Henrik Flyger ${ }^{68}$, Stig E. Bojesen ${ }^{69,70,71}$, Song Yao ${ }^{72}$, Christine B. Ambrosone ${ }^{73}$, Georgia Chenevix-Trench ${ }^{74}$, Thérèse Truong ${ }^{75,76}$, Pascal Guénel ${ }^{75,76}$, Anja Rudolph ${ }^{77}$, Jenny Chang-Claude ${ }^{77}$, Heli Nevanlinna ${ }^{78}$, Carl Blomqvist ${ }^{79}$, Kamila Czene ${ }^{15}$, Judith S. Brand ${ }^{15}$, Janet E. Olson ${ }^{80}$, Fergus J. Couch ${ }^{81}$, Alison M. Dunning ${ }^{82}$, Per Hall1 ${ }^{15}$, Douglas F. Easton ${ }^{11,82}$, Paul D. P. Pharoah ${ }^{11,82}$, Sarah E. Pinder ${ }^{1}$, Marjanka K Schmidt ${ }^{67}$, Ian Tomlinson ${ }^{83}$, Rebecca Roylance ${ }^{4}$, Montserrat García-Closas ${ }^{3,16}$ and Elinor J. Sawyer ${ }^{1 *}$

\section{Abstract}

Background: Ductal carcinoma in situ (DCIS) is a non-invasive form of breast cancer. It is often associated with invasive ductal carcinoma (IDC), and is considered to be a non-obligate precursor of IDC. It is not clear to what extent these two forms of cancer share low-risk susceptibility loci, or whether there are differences in the strength of association for shared loci.

Methods: To identify genetic polymorphisms that predispose to DCIS, we pooled data from 38 studies comprising 5,067 cases of DCIS, 24,584 cases of IDC and 37,467 controls, all genotyped using the iCOGS chip.

Results: Most (67\%) of the 76 known breast cancer predisposition loci showed an association with DCIS in the same direction as previously reported for invasive breast cancer. Case-only analysis showed no evidence for differences between associations for IDC and DCIS after considering multiple testing.

Analysis by estrogen receptor (ER) status confirmed that loci associated with ER positive IDC were also associated with ER positive DCIS. Analysis of DCIS by grade suggested that two independent SNPs at 11q13.3 near CCND1 (Continued on next page)

\footnotetext{
* Correspondence: elinor.sawyer@kcl.ac.uk

Rebecca Roylance, Montserrat García-Closas and Elinor J. Sawyer are senior co-authors

'Research Oncology, Guy's Hospital, King's College London, London, UK

Full list of author information is available at the end of the article
} 
(Continued from previous page)

were specific to low/intermediate grade DCIS (rs75915166, rs554219). These associations with grade remained after adjusting for ER status and were also found in IDC.

We found no novel DCIS-specific loci at a genome wide significance level of $P<5.0 \times 10^{-8}$.

Conclusion: In conclusion, this study provides the strongest evidence to date of a shared genetic susceptibility for IDC and DCIS. Studies with larger numbers of DCIS are needed to determine if IDC or DCIS specific loci exist.

Keywords: Ductal carcinoma in situ, Association study, Genetic predisposition, Common variants

\section{Background}

Ductal carcinoma in situ (DCIS) is a non-obligate precursor of invasive breast cancer including invasive ductal/no special type carcinomas (IDC). Since the introduction of screening mammography there has been a 7-fold increase in reported DCIS incidence in the USA, primarily in postmenopausal women [1], with about $20 \%$ of screen-detected tumors being DCIS [2]. Approximately 45-78 \% of all invasive breast cancers are associated with DCIS [3, 4]. It is hypothesized in the majority of these cases that the invasive component has arisen from the DCIS as they generally share the same somatic genetic changes. The proportion of IDC associated with DCIS varies depending on subtype, with luminal and human epidermal growth factor receptor 2 (HER2)-positive IDC having more frequent DCIS (53\% and $63 \%$, respectively) than invasive basal breast cancers (33\%) [5].

As most DCIS is treated surgically, the natural progression of untreated DCIS is not known. However, in one small study of patients with predominantly lowgrade DCIS misdiagnosed as benign breast disease and who received no surgical intervention, 6 out of $13 \mathrm{pa}-$ tients developed ipsilateral invasive carcinoma with mean time to the development of invasive carcinoma being 9.0 years [6]. In two specific DCIS trials in which DCIS was treated with breast-conserving surgery alone with no radiotherapy, long-term follow up shows that up to $30 \%$ of women develop a recurrence (half of which will be DCIS and half invasive cancer) by 10 years [7].

Methods for accurately predicting the behavior of DCIS are poor [8]. Although grade has not been shown to be a good predictor of recurrence many clinicians use this classification to determine the use of radiotherapy following breast-conserving surgery. There is a strong correlation between the grade of the in situ and coexisting invasive components in IDC, suggesting that DCIS does not progress from low through to high grade before becoming invasive $[9,10]$.

Most non-genetic risk factors for breast cancer have similar associations with DCIS and IDC, supporting the notion that DCIS is a precursor of invasive cancer [11, 12]. There is also evidence from epidemiological studies that there is an inherited predisposition to DCIS. Women with DCIS have been shown to be 2.4 times (95\% CI $0.8,7.2)$ more likely to have an affected mother and sister with breast cancer than controls [13]. Furthermore, there is evidence from a study of almost 40,000 women that the familial relative risk of DCIS is greater than that of invasive breast cancer. For women aged 30-49 years with a family history of breast cancer the odds ratio (OR) for developing DCIS was calculated as 2.4 (95 \% CI 1.1, 4.9) compared to 1.7 (95\% CI 0.9, 3.4) for invasive cancer. For women aged 50 years and above the risks were slightly reduced, but still higher for DCIS $(\mathrm{OR}=2.2,95 \% \mathrm{CI} 1.0,4.2)$ than invasive disease $(\mathrm{OR}=1.5$, $95 \%$ CI 1.0, 2.2) [14]. However, this was not confirmed in the Million Women Study, in which the association with family history was similar for DCIS and IDC [12].

A small part of this inherited predisposition is explained by BRCA1/2 mutations, as mutations in these genes are found in a similar proportion of DCIS and invasive breast cancer cases [15]. For low-risk common breast cancer predisposition alleles most of the initial breast cancer association studies have not been powered to identify associations with DCIS, so it is not clear whether all the low-risk susceptibility loci that have been identified are associated with DCIS and what the strength of any associations are.

It is now evident that some low-risk susceptibility loci are associated with different pathological subtypes of breast cancer and support the hypothesis that breast tumor subtypes arise through distinct molecular pathways [16-18]. In order to identify further low-risk susceptibility loci, it will be necessary to look at specific morphological subtypes including DCIS and the cytonuclear grade and estrogen receptor (ER) status of the disease. In this study we analyzed 3,078 cases of pure DCIS collected through the ICICLE study (a study to Investigate the genetics of In situ Carcinoma of the ductaL subtype) and performed a meta-analysis with 2,352 in situ cases collected through the Breast Cancer Association Consortium (BCAC). Our aims were to assess whether any of the known low-risk breast susceptibility alleles have different associations for DCIS and IDC, and to identify if there are any DCIS-specific low-risk alleles.

\section{Methods}

\section{Ethics statement}

All studies were performed with ethical committee approval (listed in acknowledgements) and subjects participated in the studies after providing informed consent. 


\section{Study populations}

Cases came from ICICLE (MREC 08/H0502/4), a UK study of DCIS, and from 37 studies forming part of the BCAC included in the Collaborative Oncological GeneEnvironment Study (COGS) [19] (Additional file 1). The ICICLE study recruited patients from participating centers throughout the UK with the aim of identifying predisposition genes for DCIS. Patients aged 60 years or less at the time of diagnosis, with a current or past history of DCIS (without invasive disease of any histological subtype) were eligible. A total of 3,078 subjects were recruited following identification from local pathology reports in 97 UK hospitals. All cases were genotyped with the iCOGS chip and compared to 5,000 UK controls selected from four UK studies (BBCS 1,231 controls, SBCS 704 controls, UKBGS 370 controls, SEARCH 2,695 controls) participating in BCAC (Additional file 2) and already typed on the iCOGS chip. Controls were randomly selected prior to analysis, and were excluded from casecontrol comparisons with BCAC cases from the originating study. After excluding individuals based on genotyping quality (see subsection "Genotyping and analysis") and non-European ancestry, data for the ICICLE study available for analysis included 2,715 subjects with DCIS (cases) and 4,813 controls.

Women with all types of breast cancer were recruited into the BCAC studies. Pathological information in BCAC was collected in the individual studies but was also combined and checked through standardized data control in a central database. A total of 2,352 subjects with DCIS were identified in the central BCAC pathology database (see Additional file 3 for number of cases by study). Controls came from the 37 BCAC studies (37,654 in total).

\section{Genotyping and analysis}

After DNA extraction from peripheral blood, ICICLE samples were genotyped on the iCOGS custom Illumina iSelect array (Illumina, San Diego, CA), which contains 211,155 single nucleotide polymorphisms (SNPs), at King's College London. The remaining cases and controls were genotyped as part of the COGS project described in detail elsewhere [19]. The ICICLE cases were analyzed using the same quality control $(\mathrm{QC})$ criteria as the COGS project. Briefly, genotypes were called using Illumina's proprietary GenCall algorithm and 10,000 SNPs were manually inspected to verify the algorithm calls. Individuals were excluded if genotypically nonEuropean or not female, or had an overall call rate $<95 \%$. SNPs were excluded with a Gen-Train score $<0.4$, call rate $<95 \%$ (call rate $<99 \%$ if minor allele frequency (MAF) was <0.1) and Hardy Weinberg equilibrium (HWE) value of $P<10^{-7}$ or evidence of poor clustering on inspection of cluster plots. All SNPs with MAF $<0.01$ were excluded. A cryptic relatedness analysis of the whole dataset was performed using 46,789 uncorrelated SNPs and led to the exclusion of 28 cases and 18 controls due to relatedness between the ICICLE and BCAC samples (PIHAT >0.1875).

For ICICLE cases and controls, principal component analysis (PCA) was carried out on a subset of 46,789 uncorrelated SNPs and individuals or groups distinct from the main cluster (327 cases and 164 controls) were excluded using the first five principal components (PCs) (Additional file 4). Following removal of outliers, the PCA was repeated and the first five PCs were included as covariates in the analysis.

The adequacy of the case-control matching was evaluated using quantile-quantile plots of test statistics and the inflation factor $(\lambda)$ calculated using 37,289 uncorrelated SNPs that were not selected by BCAC and were not within one of the four common fine-mapping regions, to minimize selection for SNPs associated with breast cancer (Additional file 5). As the majority of the SNPs on the iCOGS array are associated with breast, ovarian or prostate cancer, the SNPs selected for this analysis were taken from the set of prostate cancer SNPs, with the assumption that these SNPs were more likely to be representative of common SNPs in terms of population structure in our study.

For each SNP, we estimated a per-allele OR and reported corresponding $95 \% \mathrm{CI}$ using logistic regression analysis, including the five PCs as covariates, using PLINK v1.07 (http://pngu.mgh.harvard.edu/ purcell/plink/).

Genotyping and analysis of BCAC studies have been described in detail elsewhere [19]. In brief, data were analyzed using the Genotype Library and Utilities (GLU) package to estimate per-allele ORs for each SNP using unconditional logistic regression. All analyses were performed in subjects of European ancestry (determined by PC analyses) and adjusted for study and seven principal components.

Case-control ORs for DCIS cases vs controls from $\mathrm{BCAC}$ and ICICLE were combined using inverse varianceweighted fixed-effects meta-analysis, as implemented in METAL [20]. Case-only analyses were also carried out to compare genotype frequencies for (1) ER-positive (ER+) vs ER-negative (ER-) DCIS, (2) high grade DCIS vs low and intermediate grade DCIS, and (3) DCIS vs IDC (see Additional file 3 for number of cases by study), (4) DCIS diagnosis in patients $<50$ years of age vs DCIS diagnosis in patients $\geq 50$ years, and were used as a test for heterogeneity of ORs by tumor subtype/age (see Additional file 6 for number of cases by group). Only studies with data on both subtypes contributed to case-only analysis comparing these subtypes. Similar case-only analyses were performed for the IDC cases in these studies to assess whether any heterogeneity evident in DCIS also occurred in IDC. 
Novel SNPs showing the strongest evidence of association with DCIS $\left(P<6 \times 10^{-6}\right)$ in the meta-analysis (after excluding previously reported loci) were genotyped in a phase II analysis at LGC Genomics (LGC, Teddington, UK). The phase II samples consisted of 653 DCIS cases from the ICICLE and Breakthrough Generation Studies and 1,882 controls from the ICICLE study not previously genotyped on the iCOGS chip. All individuals included in the analysis were of European ancestry (self-reported).

For the known breast cancer predisposition loci $P$ $<0.00066$ was considered statistically significant (with Bonferroni correction for multiple testing on 76 known loci). All of the known breast cancer susceptibility loci were included in the iCOGS chip with the exception of rs2284378 (20q11), which was identified as an ERbreast cancer predisposition SNP after the iCOGS chip was developed [21].

\section{Assessment of grade and ER status}

For the ICICLE study, information on cytonuclear grade of DCIS was available for 2,578 cases, mostly from the local histopathology reports. In 200 cases where the grade data were missing from the report but the tumor block was available, an H\&E section was cut and the DCIS was graded by the study histopathologist (SEP) according to UK and College of American Pathologists guidelines [22]. Data on grade of DCIS were available from histopathology reports for $828 \mathrm{BCAC}$ cases.

A subset of 81 ICICLE cases, graded in the pathology report and with a tumor block available, were examined to assess the reliability of the cytonuclear grade provided by the pathology reports. In the majority of cases $(86.5 \%)$ grade was concordant with the pathology report. Nine cases were re-graded as low/intermediate grade and two cases as high grade. As the study pathologist re-graded the samples on a single $H \& E$ section, rather than all the blocks from an individual case, and in some cases on reexcision specimens with residual disease rather than the original excision specimen, the grade reported in the pathology report, if available, was used for the purposes of this study.

ER status from local histopathology reports was available for 1,086 ICICLE cases. For the remaining 781 ICICLE cases where the tumor block was available, immunohistochemistry was performed on $3-\mu \mathrm{M}$ sections, which were incubated at $60^{\circ} \mathrm{C}$ for $1 \mathrm{~h}$ prior to automated staining using the VENTANA ${ }^{\circ}$. Estrogen receptor staining was carried out using CONFIRM $^{\mathrm{su}}$ anti-estrogen receptor (SP1) rabbit monoclonal primary antibody (Catalog number 790-4324) with no variation to the recommended protocol. ER staining was scored by three independent reviewers (CP, VS, DLe) using the Allred method, and any discrepancies were reviewed by the study histopathologist
(SEP). DCIS with an Allred score $\geq 3$ was considered ER+ and DCIS with scores of 0-2 (approximately equivalent to $<1 \%$ of nuclei) was regarded as ER-. ER status was available on 965 cases from BCAC (Additional file 6).

\section{Results}

Assessment of known breast cancer susceptibility loci for association with DCIS

For the majority of known loci $(n=46)$ the risk allele for invasive breast cancer is the minor allele. For the ORs presented here the reference allele was set as the nonrisk allele to make it clear whether the association with DCIS was in the same direction as previously published for invasive breast cancer. Thus, ORs for DCIS will be $>1$ if in the same direction as invasive disease and $<1$ if in the opposite direction.

Of the 76 known common breast cancer susceptibility loci genotyped on the iCOGS array, 51 were associated with DCIS $(P<0.05)$, with the effect in the same direction as previously reported in IDC (Fig. 1 and Additional file 7). Sixteen SNPs were significantly associated with DCIS $(P<0.00066)$ with three being genome-wide significant $\left(P<5 \times 10^{-8}\right.$, Table 1$)$. The strongest associations were with for loci in FGFR2 (rs2981579: OR 1.29, $95 \%$ CI 1.24, 1.35; $P=9.0 \times 10^{-30}$ ) and TOX3 (rs3803662: OR $1.15,95 \%$ CI 1.1, $\left.1.21 ; P=1.7 \times 10^{-8}\right)$.

The case-only analysis (DCIS vs IDC) confirmed the shared genetic susceptibility between DCIS and IDC as none of the heterogeneity $P$ values $(P$-Het) were significant after Bonferroni adjustment for 76 SNPs (Additional file 7). The case-only analysis (DCIS diagnosed at $<50$ years vs $\geq 50$ years of age) revealed one SNP (rs527616, 18q11.2) that was significantly associated with DCIS in younger women $\left(P-\right.$ Het $\left._{<50 / \geq 50}=0.0003\right)$ even though the overall $P$ value for DCIS was not statistically significant after Bonferroni correction (OR 1.05, 95 \% CI 1.01, 1.11; $P=$ 0.020) (Additional file 8).

\section{Assessment of known breast cancer susceptibility loci for association with DCIS by ER status}

Following immunohistochemistry for ER in the ICICLE study samples, 1,484 cases (54\%) were classified as ER+ and 383 (14\%) as ER-. The ER data on BCAC DCIS were less complete with 664 (28\%) ER+, 301 (13\%) ER- and 1,387 cases (59 \%) of unknown ER status (Additional file 6). Analysis by ER status confirmed that loci associated with ER+ IDC were also associated with ER+ DCIS (Fig. 2 and Additional file 9). These similarities were less clear for ER- DCIS and ER- IDC but this may be due to small numbers of ER- DCIS cases. A caseonly analysis of ER+ vs ER- DCIS was not performed due to the small numbers of ER- cases. 


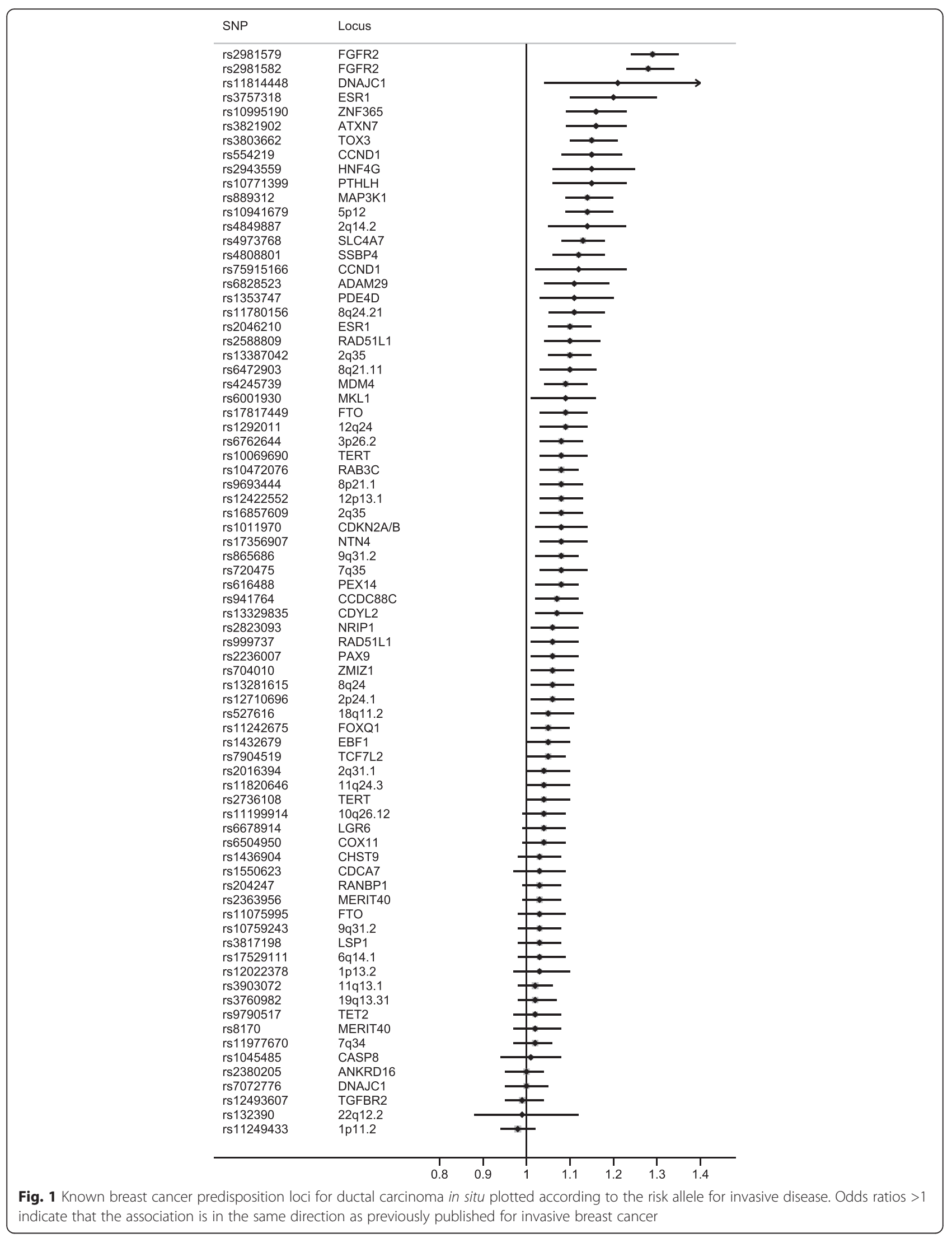


Table 1 Loci showing a significant association with ductal carcinoma in situ (DCIS) at $P<0.00066$

\begin{tabular}{|c|c|c|c|c|c|c|c|c|c|c|}
\hline \multirow[t]{2}{*}{ Chromosome } & \multirow[t]{2}{*}{ SNP } & \multirow[t]{2}{*}{ Locus } & \multirow{2}{*}{$\begin{array}{l}\text { RAF } \\
\text { Controls }\end{array}$} & \multicolumn{3}{|c|}{ DCIS vs controls (meta-analysis) } & \multicolumn{3}{|c|}{ IDC vs controls } & \multirow{2}{*}{$\begin{array}{l}\text { Case-only DCIS vs IDC } \\
\text { P-Het }\end{array}$} \\
\hline & & & & $\mathrm{OR}$ & $(95 \% \mathrm{Cl})$ & $P$ & $\mathrm{OR}$ & $(95 \% \mathrm{Cl})$ & $P$ & \\
\hline 10 & rs2981579 & FGFR2 & 0.40 & 1.29 & $(1.24,1.35)$ & $9.0 \times 10^{-30}$ & 1.24 & $(1.21,1.28)$ & $6.1 \times 10^{-66}$ & 0.14 \\
\hline 10 & rs2981582 & FGFR2 & 0.38 & 1.28 & $(1.23,1.34)$ & $1.8 \times 10^{-27}$ & 1.23 & $(1.20,1.26)$ & $2.1 \times 10^{-59}$ & 0.21 \\
\hline 16 & rs3803662 & TOX3 & 0.26 & 1.15 & $(1.10,1.21)$ & $1.7 \times 10^{-8}$ & 1.23 & $(1.20,1.27)$ & $1.5 \times 10^{-50}$ & 0.69 \\
\hline 5 & rs889312 & MAP3K1 & 0.28 & 1.14 & $(1.09,1.20)$ & $6.9 \times 10^{-8}$ & 1.11 & $(1.08,1.14)$ & $2.2 \times 10^{-14}$ & 0.13 \\
\hline 3 & rs4973768 & SLC4A7 & 0.47 & 1.13 & $(1.08,1.18)$ & $9.1 \times 10^{-8}$ & 1.09 & $(1.07,1.12)$ & $8.2 \times 10^{-13}$ & 0.58 \\
\hline 5 & rs10941679 & $5 p 12$ & 0.25 & 1.14 & $(1.09,1.20)$ & $1.3 \times 10^{-7}$ & 1.14 & $(1.11,1.18)$ & $1.2 \times 10^{-20}$ & 0.90 \\
\hline 3 & rs3821902 & ATXN7 & 0.13 & 1.16 & $(1.09,1.23)$ & $3.0 \times 10^{-6}$ & 1.06 & $(1.02,1.09)$ & 0.0030 & 0.33 \\
\hline 19 & rs4808801 & SSBP4 & 0.65 & 1.12 & $(1.06,1.18)$ & $3.1 \times 10^{-6}$ & 1.09 & $(1.05,1.11)$ & $3.5 \times 10^{-9}$ & 0.16 \\
\hline 10 & rs10995190 & ZNF365 & 0.85 & 1.16 & $(1.09,1.23)$ & $4.1 \times 10^{-6}$ & 1.15 & $(1.11,1.19)$ & $7.5 \times 10^{-16}$ & 0.61 \\
\hline 2 & rs13387042 & $2 q 35$ & 0.51 & 1.10 & $(1.05,1.15)$ & $1.1 \times 10^{-5}$ & 1.14 & $(1.11,1.16)$ & $8.3 \times 10^{-25}$ & 0.34 \\
\hline 6 & rs3757318 & ESR1 & 0.07 & 1.20 & $(1.10,1.30)$ & $1.4 \times 10^{-5}$ & 1.16 & $(1.10,1.21)$ & $1.2 \times 10^{-9}$ & 0.85 \\
\hline 11 & rs554219 & CCND1 & 0.12 & 1.15 & $(1.08,1.22)$ & $2.8 \times 10^{-5}$ & 1.27 & $(1.22,1.32)$ & $6.4 \times 10^{-38}$ & 0.88 \\
\hline 6 & rs2046210 & ESR1 & 0.34 & 1.10 & $(1.05,1.15)$ & $8.6 \times 10^{-5}$ & 1.09 & $(1.06,1.12)$ & $4.0 \times 10^{-10}$ & 0.32 \\
\hline 12 & rs10771399 & PTHLH & 0.88 & 1.15 & $(1.06,1.23)$ & 0.00021 & 1.18 & $(1.12,1.22)$ & $1.2 \times 10^{-14}$ & 0.53 \\
\hline 8 & rs11780156 & $8 q 24.21$ & 0.16 & 1.11 & $(1.05,1.18)$ & 0.00027 & 1.10 & $(1.06,1.14)$ & $2.3 \times 10^{-8}$ & 0.88 \\
\hline 16 & rs178174499 & FTO & 0.60 & 1.09 & $(1.03,1.14)$ & 0.00052 & 1.06 & $(1.04,1.10)$ & $5.9 \times 10^{-7}$ & 0.32 \\
\hline
\end{tabular}

$S N P$ single nucleotide polymorphism, IDC invasive ductal carcinoma, $O R$ odds ratio; $P$-Het $P$ value for heterogeneity; $R A F$ risk allele frequency

\section{Assessment of known breast cancer susceptibility loci for association with DCIS by grade}

Grade data were available for $95 \%$ of ICICLE DCIS cases; $1,635(60 \%)$ were of high cytonuclear grade and $943(35 \%)$ of low/intermediate grade. The grade data on the BCAC DCIS were less complete with data only available for $35 \%$ of cases: 306 (13\%) high grade and 522 (22\%) low/intermediate grade cases (Additional file 6). Case-control analysis was performed separately on the low/intermediate and high grade subsets and a case-only analysis of low/intermediate grade vs high grade DCIS was performed to assess whether any of these loci were grade-specific.

Analysis of DCIS by grade revealed that although the majority of SNPs predispose to all grades of DCIS, some are grade-specific (Additional files 10 and 11). The two SNPs close to CCND1 were strongly associated with low/intermediate grade DCIS (rs75915166, OR 1.36, $95 \%$ CI 1.17, $1.59 ; P=7.2 \times 10^{-5}$; rs554219, OR 1.32, $95 \%$ CI 1.18, 1.48; $P=8.2 \times 10^{-7}$ ) and there was no association with high grade DCIS (Table 2). Case-only analysis confirmed that these loci were low/intermediate grade-specific (rs75915166, $P$ - Het $_{\text {low } / \text { highgrade }}=0.00014$; rs554219, $P$-Het low/highgrade $=0.00013$ ) and this was independent of ER status (adjusted for ER status rs75915166, $P=0.0050$; rs554219, $P=0.019$ ).

A similar-case-only analysis of IDC by grade confirmed that the two SNPs on 11q13.3 close to CCND1 were also invasive grade 1/2-specific in IDC (rs75915166,
OR $1.42, P=1.7 \times 10^{-30}, P$-Het $=2.8 \times 10^{-10} ; \mathrm{rs} 554219$, OR 1.39, $P=4.7 \times 10^{-49}, P$-Het $\left.=1.3 \times 10^{-17}\right)$ and again were independent of ER status $\left(P=1.3 \times 10^{-6}, P=1.6 \times 10^{-6}\right.$, respectively) (Additional file 12). In addition, other gradespecific loci were identified including three (rs2363956, rs8170 and rs10069690) specific to grade 3 invasive disease (Additional file 13).

rs10941679, 5p12 were borderline associated with low/intermediate grade DCIS (OR 1.26, $P=2.1 \times 10^{-7}$, $P$-Het low $/$ highgrade $=0.0033$ ). This locus has previously been shown to be associated with low grade progesterone receptor (PR) + IDC [23]. There was no evidence of any high grade DCIS specific loci (Additional file 11).

\section{Search for new DCIS predisposition loci}

All SNPs that were genome-wide significant $\left(P<5 \times 10^{-8}\right)$ in the meta-analysis were correlated with one of the known breast cancer predisposition loci. There were three SNPs that were not correlated with known loci at $P<6 \times$ $10^{-6}$ (Table 3 ), all with very little evidence of an association with IDC.

Of these novel SNPs, rs12631593, 3p14.2, (an intronic variant in FHIT, chr3: 60726844) was the most strongly associated with DCIS (OR 1.21, $95 \%$ CI 1.13, 1.29; $P=$ $\left.5.5 \times 10^{-8}\right)$. This SNP showed little association with IDC (OR 1.01, $95 \%$ CI 0.97, 1.05; $P=0.54$ ) and this was supported by the case-only analysis $\left(P\right.$-Het ${ }_{\text {DCIS/IDC }}=$ 0.0048). 

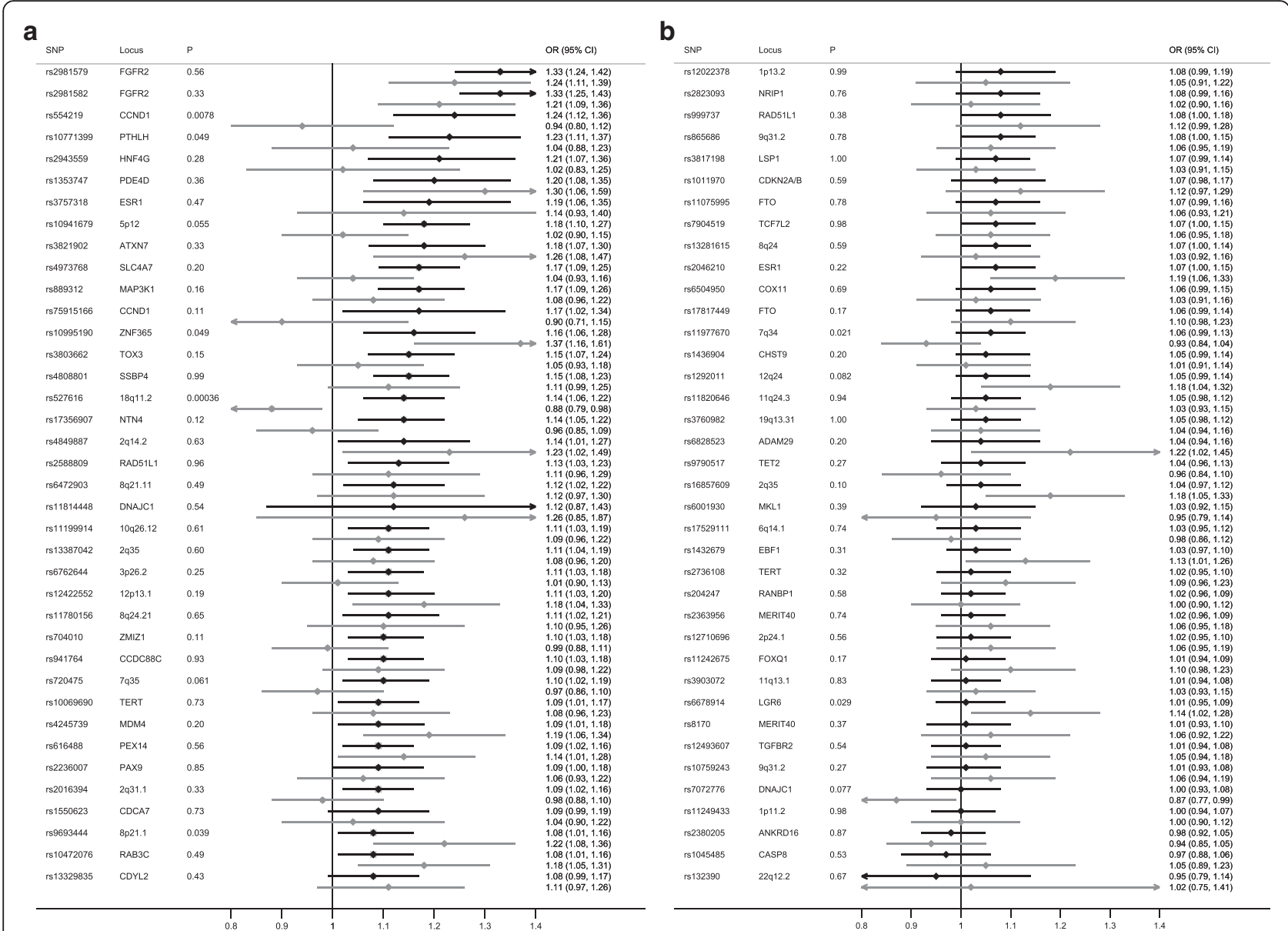

Fig. 2 Known breast cancer predisposition loci for estrogen receptor-positive (ER+) (black lines) and ER- ductal carcinoma in situ (gray lines). Due to the large number of single nucleotide polymorphisms (SNPS), for better visual representation the plot is split into two different sections (a and $\mathbf{b}$ ) with a descending order of effect size for the ER+ group. OR odds ratio

The other loci were on 22q13.2, rs73179023 (DCIS only: OR $0.85,95 \%$ CI $0.79,0.90 ; P=1.1 \times 10^{-6}$; IDC only: OR 0.97, $95 \%$ CI 0.93, 1.00; $P=0.060, P$-Het $\mathrm{DCIS} / \mathrm{IDC}=0.0099$ ) and 7q21.3, rs13236351 (DCIS only: OR 1.30, $95 \%$ CI 1.16, 1.46; $P=5.7 \times 10^{-6}$; IDC only: OR $1.05,95 \%$ CI 0.99 , $1.13 ; P=0.13, P-$ Het $\left._{\text {DCIS } / \text { IDC }}=0.17\right)$.

These SNPs were genotyped in a validation study including a further 653 DCIS cases and 1,882 controls, however, for all three loci there was no evidence of an association (for rs12631593, rs13236351, and rs73179023, $P=0.49,0.61$, and 0.57 , respectively) and none were genome wide significant following a meta-analysis of all data $\left(P=7.8 \times 10^{-7}, 2.9 \times 10^{-5}\right.$, and $1.7 \times 10^{-6}$ respectively $)$ (Table 3).

\section{Discussion}

This study provides the strongest evidence to date for a shared genetic susceptibility between DCIS and IDC, based on 5,067 cases with pure DCIS (no invasive disease) and 24,670 cases with IDC. It differs from previous BCAC analyses of DCIS, as it has included an additional 3,078 DCIS cases, excluded all cases of pure LCIS and has also compared DCIS to IDC rather than all invasive disease.

An important finding of this study is the lack of DCIS/ IDC-specific loci among the known breast cancer predisposition loci. Of the five breast cancer predisposition alleles originally reported by Easton et al. [24], three were shown to be associated with in situ (998 cases of DCIS and LCIS) disease (rs2981582-FGFR2, rs3803662TOX3, rs889312-MAP3K1) with rs889312 showing a stronger association with DCIS ( $P$-trend 0.007 , per allele OR 1.30 for DCIS, per allele OR 1.13 for invasive disease). However, this finding of potential DCIS-specific loci was not confirmed in the Million women study which found no differential association with DCIS vs IDC for twelve breast cancer susceptibility loci, including rs889312, although their sample size was smaller (873 DCIS and 4,959 IDC) [12]. In the recent BCAC 
Table 2 Association between rs75915166 or rs554219 and grade in ductal carcinoma in situ

\begin{tabular}{|c|c|c|c|c|c|}
\hline & \multicolumn{5}{|l|}{ Meta-analysis } \\
\hline & OR (95\% Cl) & $P$ & $\begin{array}{l}\text { Low/intermediate grade, } \\
\text { number }\end{array}$ & $\begin{array}{l}\text { High grade, } \\
\text { number }\end{array}$ & Controls, number \\
\hline \multicolumn{6}{|l|}{ rs75915166 } \\
\hline Low/intermediate grade vs controls & $1.36(1.17,1.59)$ & $7.2 \times 10^{-5}$ & 1,465 & & 35,521 \\
\hline High grade vs controls & $0.92(0.79,1.08)$ & 0.31 & & 1,941 & 32,202 \\
\hline \multicolumn{6}{|l|}{ Case-only high vs low/intermediate grade } \\
\hline Unadjusted & $0.68(0.55,0.83)$ & $1.4 \times 10^{-4}$ & 1,307 & 1,941 & \\
\hline unadjusted (only cases with ER status) & $0.65(0.51,0.84)$ & $1.1 \times 10^{-3}$ & 791 & 1,360 & \\
\hline adjusted for ER status & $0.68(0.52,0.89)$ & 0.0050 & 791 & 1,360 & \\
\hline ER+ only & $0.68(0.55,0.84)$ & $5 \times 10^{-4}$ & 709 & 985 & \\
\hline \multicolumn{6}{|l|}{ rs554219 } \\
\hline Low/intermediate grade vs controls & $1.32(1.18,1.48)$ & $8.2 \times 10^{-7}$ & 1,465 & & 35,521 \\
\hline High grade vs controls & $1.02(0.91,1.14)$ & 0.75 & & 1,941 & 32,202 \\
\hline \multicolumn{6}{|l|}{ Case-only high vs low/intermediate grade } \\
\hline Unadjusted & $0.75(0.65,0.87)$ & $1.3 \times 10^{-4}$ & 1,307 & 1,941 & \\
\hline unadjusted (only cases with ER status) & $0.75(0.63,0.88)$ & $2.1 \times 10^{-4}$ & 791 & 1,360 & \\
\hline adjusted for ER status & $0.80(0.67,0.96)$ & 0.019 & 792 & 1,360 & \\
\hline ER+ only & $0.76(0.65,0.89)$ & $6.7 \times 10^{-4}$ & 709 & 985 & \\
\hline
\end{tabular}

$O R$ odds ratio, $E R$ estrogen receptor

COGS analysis all 41 novel SNPs identified on the iCOGS chip had comparable ORs for invasive and in situ disease (based on data from 2,335 in situ, and 42,118 invasive cases), with the exceptions of rs12493607 (TGFBR2), and rs3903072 (11q13.1), for which associations seemed to be restricted to invasive disease [19]; however, we found no evidence of an IDC-specific association with these loci after correcting for multiple testing. A recent study investigating the association between 39 of the known breast cancer predisposition loci and breast cancer in situ (BCIS) suggested that rs1011970 (9p21.3, CDKN2BAS) had a stronger association with BCIS than invasive breast cancer (BC), $P$-Het HCIS $/ \mathrm{BC}=0.0065$. This trend remained in a DCIS vs $\mathrm{BC}$ analysis $\left(P-\right.$ Het $\left._{\mathrm{DCIS} / \mathrm{BC}}=0.021\right)[25]$. Our data, however, do not support this finding (DCIS OR 1.08, $95 \%$ CI 1.02, 1.14; $P=0.011$; IDC OR 1.05, $95 \%$ CI 1.0, 1.09; $P=0.0025, P$-Het ${ }_{\text {DCIS } / \text { IDC }}=0.33$ ).

We have also shown for the first time that seven of the known invasive breast cancer predisposition loci not previously shown to be associated with DCIS have comparable ORs for IDC and DCIS: rs4973768 (SLC4A7), rs3821902 (ATXN7) [26], rs109 95190 (ZNF365), rs554219 (CCND1), rs3757318 and rs2046210 (ESR1).

This lack of DCIS/IDC-specific loci is in contrast to our previous study of lobular cancer in which we showed that there are loci that are specific to invasive lobular cancer (ILC), showing no association with lobular carcinoma in situ (LCIS) and there was also a suggestion of LCISspecific loci [16]. When we compare the DCIS data presented here to our previous LCIS analyses it reveals that there is some overlap between loci that are associated with ER+ DCIS and LCIS (Fig. 3 and Additional file 14). However, there are also some differences: rs6678914, LGR6 and rs865686, 9q31.2 are strongly associated with LCIS but there is little evidence of association with ER+ DCIS $\left(P\right.$-Het DCIS/LCIS $=7.4 \times 10^{-5}$ and $6.6 \times 10^{-4}$, respectively $)$. We have also previously shown that rs11249433, 1p11.2 and rs11977670, 7q34 have a stronger association with invasive lobular cancer than IDC [16]. These loci were only weakly associated with LCIS and were not associated with ER+ DCIS in this analysis.

Most association studies of invasive breast cancer involve subgroup analyses based on ER status. In contrast to invasive breast cancer, ER status in DCIS is not routinely assessed in all centers despite evidence from the NSABP B-24 trial of benefit from endocrine therapy in ER+ DCIS [7]. A national audit of DCIS in the UK revealed that ER status was assessed in only $50 \%$ of DCIS cases and ER positivity in low and intermediate grade DCIS was significantly more common than in high grade DCIS $(P<0.001)(\mathrm{ER}+$ high grade $69 \%$, intermediate grade $94 \%$, low grade $99 \%$ ) [27]. In order to overcome this issue we performed ER immunohistochemistry on the samples from ICICLE for which ER status was unknown. However, there was still a large amount of 
Table 3 Potential new ductal carcinoma in situ susceptibility loci

\begin{tabular}{|c|c|c|c|}
\hline Single nucleotide polymorphism & rs12631593 & rs13236351 & rs73179023 \\
\hline Chromosome & 3 & 7 & 22 \\
\hline Position & 60701884 & 97772513 & 43424477 \\
\hline Locus & FHIT & LMTK2 & PACSIN2:TTLL1 \\
\hline Minor allele frequency & 0.11 & 0.032 & 0.13 \\
\hline \multicolumn{4}{|l|}{ ICICLE DCIS phase I } \\
\hline Odds ratio $(95 \% \mathrm{Cl})$ & $1.15(1.04,1.28)$ & $1.31(1.10,1.56)$ & $0.83(0.75,0.91)$ \\
\hline$P$ & 0.0088 & 0.0029 & 0.00020 \\
\hline \multicolumn{4}{|l|}{ BCAC DCIS } \\
\hline Odds ratio $(95 \% \mathrm{Cl})$ & $1.25(1.14,1.36)$ & $1.3(1.12,1.51)$ & $0.86(0.79,0.94)$ \\
\hline$P$ & $1.0 \times 10^{-6}$ & 0.00060 & 0.0012 \\
\hline \multicolumn{4}{|l|}{ Meta-analysis phase I } \\
\hline Odds ratio (95% Cl) & $1.21(1.13,1.29)$ & $1.3(1.16,1.46)$ & $0.85(0.79,0.90)$ \\
\hline$P$ & $5.5 \times 10^{-8}$ & $5.7 \times 10^{-6}$ & $1.1 \times 10^{-6}$ \\
\hline \multicolumn{4}{|l|}{ Phase II DCIS } \\
\hline Odds ratio (95% Cl) & $0.93(0.76,1.14)$ & $0.91(0.63,1.31)$ & $0.95(0.78,1.15)$ \\
\hline$P$ & 0.49 & 0.61 & 0.57 \\
\hline \multicolumn{4}{|l|}{ Meta-analysis phase II } \\
\hline Odds ratio $(95 \% \mathrm{Cl})$ & $1.18(1.10,1.25)$ & $1.26(1.13,1.41)$ & $0.86(0.80,0.91)$ \\
\hline$P$ & $7.8 \times 10^{-7}$ & $2.9 \times 10^{-5}$ & $1.7 \times 10^{-6}$ \\
\hline \multicolumn{4}{|l|}{$\mathrm{BCAC}$ IDC } \\
\hline Odds ratio $(95 \% \mathrm{Cl})$ & $1.01(0.97,1.05)$ & $1.05(0.99,1.13)$ & $0.97(0.93,1.00)$ \\
\hline$P$ & 0.54 & 0.13 & 0.060 \\
\hline \multicolumn{4}{|l|}{ Case-only } \\
\hline DCIS vs IDC $P$-Het & 0.0048 & 0.17 & 0.0099 \\
\hline
\end{tabular}

DCIS ductal carcinoma in situ, IDC invasive ductal carcinoma, BCAC Breast Cancer Association Consortium, ICICLE Study to investigate the genetics of in situ carcinoma of the ductal subtype, $P$-Het $P$ value for heterogeneity

missing data on ER status in the BCAC cases, resulting in only 684 ER- DCIS cases being available for analysis, making it difficult to draw definitive conclusions about ER- DCIS. In essence the findings are similar to invasive breast cancer, with ER- and ER+ DCIS having different genetic susceptibility profiles and ER+ DCIS having a very similar profile to ER+ IDC.

Cytonuclear grade of DCIS is used by many clinicians to select those cases most likely to benefit from radiotherapy despite the fact that grade has not been shown to be a good predictor of recurrence. In the UK audit of DCIS, grade data were available for $99 \%$ of DCIS cases, with $59 \%$ classified as high grade, $29 \%$ as intermediate and $11 \%$ as low grade [27]. Similarly, in our study data on grade were available for $95 \%$ of cases in ICICLE. In invasive disease only a minority of predisposition loci have been shown to be grade specific; rs2981582 (FGFR2) and rs13281615 (8q24) [28, 29] and rs10941679 (5p12) [23]. We have shown that analysis of DCIS by grade reveals other known loci that are grade specific. The loci with the strongest association with grade were SNPs on 11q13, which had a stronger association with low/intermediate grade DCIS and IDC than high grade lesions. The finding of a strong association with low and intermediate grade ductal carcinomas that is independent of ER status in both DCIS and IDC for these loci is novel. rs614367 was the first locus on 11q13 shown to be associated with invasive breast cancer [30]. Fine mapping of the region subsequently identified two independent signals (rs554219 and rs78540526, $r^{2}=0.38$ ), which are the loci reported in this analysis. Functional analyses demonstrated that the risk variants modify enhancer and silencer elements, with the likely target gene being CCND1 [31].

A study of 150 cases of subsequent breast cancer (invasive and in situ) after DCIS observed significant association for both grade and ER status between the index DCIS and the subsequent breast cancer (whether ipsilateral or contralateral), suggesting that women with DCIS are at risk of developing subsequent breast cancers 


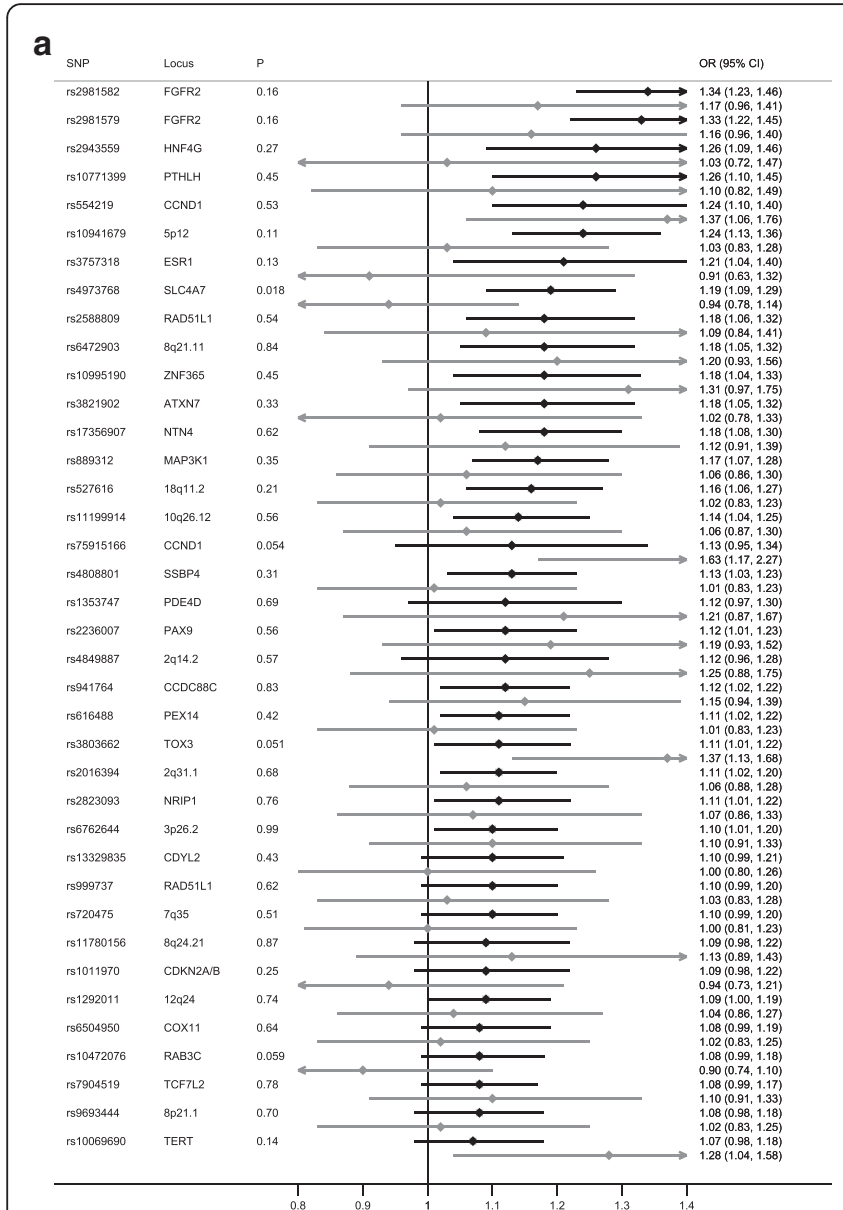

b

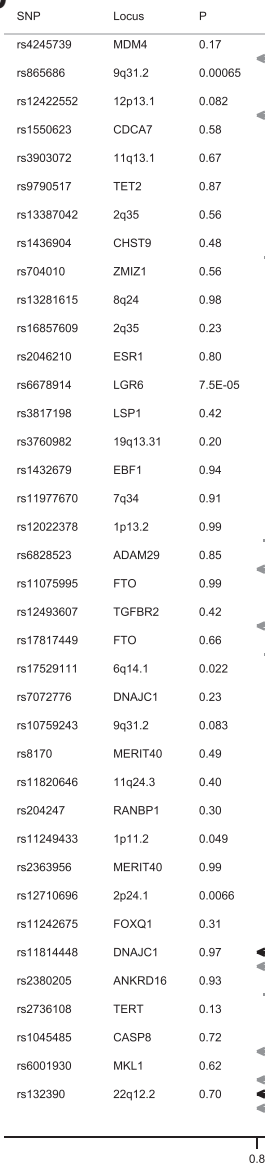

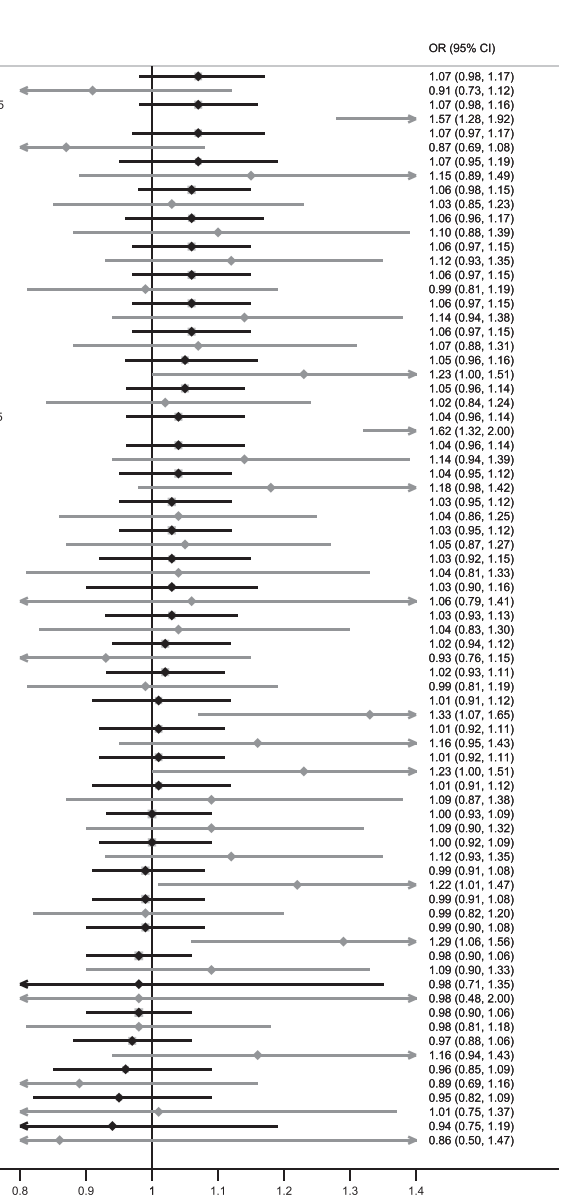

Fig. 3 Known breast cancer predisposition loci for estrogen receptor-positive (ER+) (black) ductal carcinoma in situ and lobular carcinoma in situ (gray). Due to the large number of single nucleotide polymorphisms (SNPs), for better visual representation, the plot is split into two different sections (a and $\mathbf{b}$ ) with a descending order of effect size for the ER+ group. OR odds ratio

of a similar phenotype [32]. This finding supports the genetic predisposition data presented here, with ER and grade-specific loci in DCIS having similar specificity in IDC.

Although we did not identify any novel loci that reached genome wide significance, we did identify three potential novel DCIS predisposition loci, two of which were DCIS-specific (rs12631593, rs73179023), and therefore need further investigation in other cohorts of DCIS. As at least $45 \%$ of patients with IDC have associated DCIS present at diagnosis consistent with direct precursor behavior, it may seem biologically implausible that an SNP predisposes to DCIS but is not associated with IDC. However, it is possible that there is a subset of patients with DCIS with very low probability of progression. If the finding of DCIS-specific predisposition loci were confirmed in other studies, identifying such a subset of patients with low-risk DCIS would be clinically valuable.

\section{Conclusion}

In conclusion this is the largest study to assess genetic predisposition in DCIS and shows that the majority of invasive breast cancer predisposition loci also predispose to DCIS. It highlights that, as for invasive disease, different SNPs predispose to ER+ and ER- DCIS. In addition it shows the importance of grade in both DCIS and IDC.

\section{Additional files}

Additional file 1: Study information for the Breast Cancer Association Consortium (BCAC) participating studies. (DOCX $28 \mathrm{~kb}$ )

Additional file 2: Sample information for the SEARCH, UKBGS, SBCS, and BBCS studies. (DOCX $15 \mathrm{~kb}$ )

Additional file 3: Number of studies and individuals included in analyses of ductal carcinoma in situ (DCIS) and invasive ductal carcinoma (IDC). BCAC Breast Cancer Association Consortium. (XLSX 12 kb)

Additional file 4: Principal component analysis (PCA) results from the study to investigate the genetics of in situ carcinoma of the ductal subtype (ICICLE). (PPTX $142 \mathrm{~kb}$ ) 
Additional file 5: Quantile-quantile plots from the study to investigate the genetics of in situ carcinoma of the ductal subtype (ICICLE). SNP single nucleotide polymorphism. (PPTX $125 \mathrm{~kb}$ )

Additional file 6: Grade, estrogen receptor (ER) status, and age groups in patients with ductal carcinoma in situ (DCIS). BCAC Breast Cancer Association Consortium, ICICLE study to investigate the genetics of in situ carcinoma of the ductal subtype. (DOCX $16 \mathrm{~kb}$ )

Additional file 7: Association between ductal carcinoma in situ (DCIS) and known breast cancer predisposition loci. IDC invasive ductal carcinoma, $P$-Het $P$ value for heterogeneity, SNP single nucleotide polymorphism, OR odds ratio. (XLSX $19 \mathrm{~kb}$ )

Additional file 8: Age-specific case-only analysis of patients with ductal carcinoma in situ (DCIS) diagnosed at age $<\mathbf{5 0}$ vs $\geq \mathbf{5 0}$ years. $P$-Het $P$ value for heterogeneity, SNP single nucleotide polymorphism, $O R$ odds ratio. (XLSX $17 \mathrm{~kb}$ )

Additional file 9: Associations between the known breast cancer predisposition loci and estrogen receptor-positive $(E R+)$ or ER- ductal carcinoma in situ (DCIS). P-Het $P$ value for heterogeneity, SNP single nucleotide polymorphism, OR odds ratio. (XLSX $19 \mathrm{~kb}$ )

Additional file 10: $a$, $b$ Known breast cancer predisposition loci for low/intermediate grade (black) and high grade ductal carcinoma in situ (DCIS) (gray). Due to the large number of single nucleotide polymorphisms (SNPS), the plot is split for better visual representation into two different sections ( $a$ and $b$ ) with a descending order of effect size for the low/intermediate group. OR odds ratio. (ZIP $20 \mathrm{~kb}$ )

Additional file 11: Associations of the known breast cancer predisposition loci for high and low-intermediate grade ductal carcinoma in situ (DCIS). $P$-Het $P$ value for heterogeneity, OR odds ratio. (XLSX $18 \mathrm{~kb})$

Additional file 12: Association of rs75915166 and rs554219 with grade in invasive ductal carcinoma (IDC). $P$-Het $P$ value for heterogeneity, OR odds ratio. (XLSX 9 kb)

Additional file 13: Associations between the known and novel breast cancer predisposition loci and invasive ductal cancer, by estrogen receptor (ER) status and grade. OR odds ratio. (XLSX $24 \mathrm{~kb}$ )

Additional file 14: Association between the known breast cancer predisposition loci and estrogen receptor-positive $(E R+)$ ductal carcinoma in situ (DCIS) or lobular carcinoma in situ (LCIS). P-Het $P$ value for heterogeneity, SNP single nucleotide polymorphism, OR odds ratio. (XLSX $19 \mathrm{~kb})$

\section{Abbreviations}

ABCS: Amsterdam Breast Cancer Study; BBBC: Bavarian Breast Cancer Cases and Controls; BBCS: British Breast Cancer Study; BC: breast cancer; BCAC: Breast Cancer Association Consortium; BCIS: breast carcinoma in situ; Cl: confidence interval; COGS: Collaborative Oncological Gene-Environment Study; DCIS: ductal carcinoma in situ; ER: Estrogen receptor; H\&E: hematoxylin and eosin; HWE: Hardy Weinberg equilibrium; ICICLE: study to investigate the genetics of in situ carcinoma of the ductal subtype; IDC: invasive ductal carcinoma; LCIS: Iobular carcinoma in situ; MAF: minor allele frequency; OR: odds ratio; PCA: principal component analysis; $P$-Het: $P$ value for heterogeneity; SNP: single nucleotide polymorphism.

\section{Competing interests}

The authors declare that there are no conflicts of interest.

\section{Authors' contributions}

The study was conceived by ES and RR. Analysis and genotyping in ICICLE was performed by ES. Meta-analyses were performed by MGC. The manuscript was prepared by ES. EJS, RR and IT conceived and designed the experiments. CP, VS, DLe, EP, AGN, DCT, DV, FB, JD, and AMD performed the experiments. CP, MNB, MKB, QW, KM, IT, MGC, and EJS analyzed the data. CP, MNB, VS, KK, PGo, MC, DLe, EP, NO, AC, SSC, IdSS, JP, AS, MJS, MKB, QW, JD, KM, JB, AGN, DCT, DV, JLi, JF, VK, ALBD, PS, JS, RLM, GGG, SM, AL, TB, HBra, MCS, JLH, TD, NVB, MK, UH, RKS, AMe, HBre, VA, RW, KP, PAF, MWB, JLu, AJ, AMM, ILA, RAEMT, PD, LLM, CAH, AMa, VMK, PR, PP, FM, BB, CHMvD, AH, NM,
MJK, DLa, GF, JW, HF, SEB, SY, CBA, GCT, TT, PGu, AR, JCC, HN, CB, KC, JSB, JEO, FJC, AMD, PH, DFE, PDPP, SEP, MKS, IT, RR, MGC, and EJS contributed reagents/materials/analysis tools. CP, IT, MGC, and EJS wrote the paper. SEP performed the histopathology review. CP, VS, DLe, and SEP performed ER scoring. CP, MNB, VS, KK, PGO, MC, DLe, EP, NO, AC, SSC, IdSS, JP, AS, MJS, $M K B, Q W, J D, K M, J B, A G N, D C T, D V$, JLi, JF, VK, ALBD, PS, JS, RLM, GGG, SM, AL, TB, HBra, MCS, JLH, TD, NVB, MK, UH, RKS, AMe, HBre, VA, RW, KP, PAF, MWB, JLu, AJ, AMM, ILA, RAEMT, PD, LLM, CAH, AMa, VMK, PR, PP, FM, BB, CHMvD, AH, NM, MJK, DLa, GF, JW, HF, SEB, SY, CBA, GCT, TT, PGu, AR, JCC, $H N, C B, K C$, JSB, JEO, FJC, AMD, PH, DFE, PDPP, SEP, MKS, IT, RR, MGC, and EJS provided critical review of the manuscript. CP, MNB, VS, KK, PGo, MC DLe, EP, NO, AC, SSC, IdSS, JP, AS, MJS, MKB, QW, JD, KM, JB, AGN, DCT, DV, JLi, JF, VK, ALBD, PS, JS, RLM, GGG, SM, AL, TB, HBra, MCS, JLH, TD, NVB, MK, UH, RKS, AMe, HBre, VA, RW, KP, PAF, MWB, JLu, AJ, AMM, ILA, RAEMT, PD, LLM, CAH AMa VMK PR PP FM BB CHMVD AH NM MJK DLa GF JW HF SEB SY CBA GCT TT, PGu, AR, JCC, HN, CB, KC, JSB, JEO, FJC, AMD, PH, DFE, PDPP, SEP, MKS, IT, RR, MGC, and EJS approved the final version of the manuscript.

\section{Authors' information}

Study was conceived by ES \& RR, analysis \& genotyping of ICICLE performed by ES, meta-analyses performed by MGC, manuscript prepared by ES.

\section{Acknowledgements}

We thank all the individuals who took part in these studies and all the researchers, clinicians, technicians and administrative staff who have enabled this work to be carried out. This study would not have been possible without the contributions of the following: Andrew Berchuck (OCAC), Rosalind A. Eeles, Ali Amin Al Olama, Zsofia Kote-Jarai, Sara Benlloch (PRACTICAL), Antonis Antoniou, Lesley McGuffog, Ken Offit (CIMBA), Andrew Lee, and Ed Dicks, Craig Luccarini and the staff of the Centre for Genetic Epidemiology Laboratory, the staff of the CNIO genotyping unit, Francois Bacot, Sylvie LaBoissière and Frederic Robidoux and the staff of the McGill University and Génome Québec Innovation Centre, Sune F. Nielsen, Borge G. Nordestgaard, and the staff of the Copenhagen DNA Laboratory, and Julie M. Cunningham, Sharon A. Windebank, Christopher A. Hilker, Jeffrey Meyer and the staff of Mayo Clinic Genotyping Core Facility. In particular, we thank: Maria Troy (ICICLE); the Swedish Medical Research Council (pKARMA); Siranoush Manoukian, Bernard Peissel, Daniela Zaffaroni and Jacopo Azzollini of the Fondazione IRCCS Istituto Nazionale dei Tumori (INT); Bernado Bonanni, Monica Barile and Irene Feroce of the Istituto Europeo di Oncologia (IEO), and the personnel of the Cogentech Cancer Genetic Test Laboratory (MBCSG); Emily Hallberg for contributions to sample and phenotype management (MCBCS); the SEARCH and EPIC teams, Kirsimari Aaltonen, Karl von Smitten, Sofia Khan, Tuomas Heikkinen, Irja Erkkilä (HEBCS); Petra Seibold, Dieter Flesch-Janys, Judith Heinz, Nadia Obi, Alina Vrieling, Sabine Behrens, Ursula Eilber, Muhabbet Celik, Til Olchers and Stefan Nickels (MARIE); Eileen Williams, Elaine Ryder-Mills, Kara Sargus (BBCS); Heather Thorne, Eveline Niedermayr, all the kConFab research nurses and staff, the heads and staff of the Family Cancer Clinics, and the Clinical Follow Up Study (which has received funding from the NHMRC, the National Breast Cancer Foundation, Cancer Australia, and the National Institute of Health (USA)) for their contributions to this resource, and the many families who contribute to kConFab; members of the Data Bank and Biorepository (DBBR) at Roswell Park Cancer Institute (RPCI) for providing biospecimens; staff and participants of the Copenhagen General Population Study and for the excellent technical assistance of Dorthe Uldall Andersen, Maria Birna Arnadottir, Anne Bank, Dorthe Kjeldgård Hansen (CGPS); Sten Cornelissen, Richard van Hien, Linde Braaf, Frans Hogervorst, Senno Verhoef, Laura van 't Veer, Emiel Rutgers, C Ellen van der Schoot, Femke Atsma (ABCS); Sue Higham, Helen Cramp, lan Brock, Sabapathy Balasubramanian, Malcolm W.R. Reed and Dan Connley (SBCS); Breakthrough Breast Cancer and the Institute of Cancer Research for support and funding of the Breakthrough Generations Study, and the study participants, study staff, and the doctors, nurses and other health care providers and health information sources who have contributed to the study and acknowledge NHS funding to the Royal Marsden/ICR NIHR Biomedical Research Centre; Gilian Peuteman, Dominiek Smeets, Thomas Van Brussel and Kathleen Corthouts (LMBC); Niall Mclnerney, Gabrielle Colleran, Andrew Rowan, Angela Jones (BIGGS); Petra Bos, Jannet Blom, Ellen Crepin, Elisabeth Huijskens, Annette Heemskerk, the Erasmus MC Family Cancer Clinic (RBCS): Peter Bugert, Medical Faculty Mannheim (BSUCH); Eija Myöhänen, Helena 
Kemiläinen (KBCP); E. Krol-Warmerdam, and J. Blom for patient accrual, administering questionnaires, and managing clinical information, the LUMC survival data were retrieved from the Leiden hospital-based cancer registry system (ONCDOC) with the help of Dr. J. Molenaar (ORIGO); Guillermo Pita, Charo Alonso, Daniel Herrero, Nuria Álvarez, Pilar Zamora, Primitiva Menendez, the Human Genotyping-CEGEN Unit (CNIO); Teresa Selander, Nayana Weerasooriya (OFBCR); Arja Jukkola-Vuorinen, Mervi Grip, Saila Kauppila; Kari Mononen and Meeri Otsukka (OBCS); Hartwig Ziegler, Sonja Wolf, Christa Stegmaier, Katja Butterbach, Stefanie Engert, Heide Hellebrand, Sandra Kröber, Peter Hillemanns, Hans Christiansen and Johann H. Karstens (HMBCS); Maggie Angelakos, Judi Maskiell, Gillian Dite (ABCFS); The GENICA Network: Dr. Margarete Fischer-Bosch-Institute of Clinical Pharmacology, Stuttgart, and University of Tübingen, Germany [HB, Wing-Yee Lo, Christina Justenhoven], German Cancer Consortium (DKTK) and German Cancer Research Center (DKFZ) (HB), Department of Internal Medicine, Evangelische Kliniken Bonn gGmbH, Johanniter Krankenhaus, Bonn, Germany (Yon-Dschun Ko, Christian Baisch), Institute of Pathology, University of Bonn, Germany (Hans-Peter Fischer), Molecular Genetics of Breast Cancer, Deutsches Krebsforschungszentrum (DKFZ), Heidelberg, Germany (Ute Hamann), Institute for Prevention and Occupational Medicine of the German Social Accident Insurance, Institute of the Ruhr University Bochum (IPA), Bochum, Germany (TB, Beate Pesch, Sylvia Rabstein, Anne Lotz); and Institute of Occupational Medicine and Maritime Medicine, University Medical Center Hamburg-Eppendorf, Germany (Volker Harth); Martine Tranchant (CHU de Québec Research Center), Marie-France Valois, Annie Turgeon and Lea Heguy (McGill University Health Center, Royal Victoria Hospital; McGill University) for DNA extraction, sample management and skillful technical assistance. JS is Chairholder of the Canada Research Chair in Oncogenetics (MTLGEBCS); Dr. Kristine Kleivi, PhD (K.G. Jebsen Centre for Breast Cancer Research, Institute of Clinical Medicine, University of Oslo, Oslo, Norway and Department of Research, Vestre Viken, Drammen, Norway), Dr. Lars Ottestad, MD (Department of Genetics, Institute for Cancer Research, Oslo University Hospital Radiumhospitalet, Oslo, Norway), Prof. Em. Rolf Kåresen, MD (Department of Oncology, Oslo University Hospital and Faculty of Medicine, University of Oslo, Oslo, Norway), Dr. Anita Langerød, PhD (Department of Genetics, Institute for Cancer Research, Oslo University Hospital Radiumhospitalet, Oslo, Norway), Dr. Ellen Schlichting, MD (Department for Breast and Endocrine Surgery, Oslo University Hospital Ullevaal, Oslo, Norway), Dr. Marit Muri Holmen, MD (Department of Radiology and Nuclear Medicine, Oslo University Hospital, Oslo, Norway), Prof. Toril Sauer, MD (Department of Pathology at Akershus University hospital, Lørenskog, Norway), Dr. Vilde Haakensen, MD (Department of Genetics, Institute for Cancer Research, Oslo University Hospital Radiumhospitalet, Oslo, Norway), Dr. Olav Engebråten, MD (Institute for Clinical Medicine, Faculty of Medicine, University of Oslo and Department of Oncology, Oslo University Hospital, Oslo, Norway), Prof. Bjørn Naume, MD (Division of Cancer Medicine and Radiotherapy, Department of Oncology, Oslo University Hospital Radiumhospitalet, Oslo, Norway), Dr. Cecile E. Kiserud, MD (National Advisory Unit on Late Effects after Cancer Treatment, Department of Oncology, Oslo University Hospital, Oslo, Norway and Department of Oncology, Oslo University Hospital, Oslo, Norway), Dr. Kristin V. Reinertsen, MD (National Advisory Unit on Late Effects after Cancer Treatment, Department of Oncology, Oslo University Hospital, Oslo, Norway and Department of Oncology, Oslo University Hospital, Oslo, Norway), Assoc. Prof. Åslaug Helland, MD (Department of Genetics, Institute for Cancer Research and Department of Oncology, Oslo University Hospital Radiumhospitalet, Oslo, Norway), Dr. Margit Riis, MD (Dept of Breastand Endocrine Surgery, Oslo University Hospital, Ullevål, Oslo, Norway), Dr. Ida Bukholm, MD (Department of Breast-Endocrine Surgery, Akershus University Hospital, Oslo, Norway and Department of Oncology, Division of Cancer Medicine, Surgery and Transplantation, Oslo University Hospital, Oslo, Norway), Prof. Per Eystein Lønning, MD (Section of Oncology, Institute of Medicine, University of Bergen and Department of Oncology, Haukeland University Hospital, Bergen, Norway), Dr Silje Nord, PhD (Department of Genetics, Institute for Cancer Research, Oslo University Hospital Radiumhospitalet, Oslo, Norway) and Grethe I. Grenaker Alnæs, M.Sc. (Department of Genetics, Institute for Cancer Research, Oslo University Hospital Radiumhospitalet, Oslo, Norway) (NBCS); Louise Brinton, Mark Sherman, Neonila Szeszenia-Dabrowska, Beata Peplonska, Witold Zatonski, Pei Chao, and Michael Stagner (PBCS). kConFab/ AOCS Investigators (Georgia.Trench@qimrberghofer.edu.au) Peter MacCallum Cancer Center, The University of Melbourne, Melbourne, Australia. Funding was as follows: ICICLE genotyping was funded by the Breast Cancer Now (http://breastcancernow.org/), and sample and data collection by Cancer Research UK. Core funding came from the National Institute for Health Research (NIHR) Biomedical Research Centre at Guy's and St. Thomas' NHS Foundation Trust and King's College London and the Wellcome Trust Centre for Human Genetics (provided by the Wellcome Trust, 090532/Z/09/ Z). The views expressed are those of the author(s) and not necessarily those of the NHS, NIHR or the Department of Health. BCAC is funded by Cancer Research UK (C1287/A10118, C1287/A12014) and by the European Community's Seventh Framework Programme under grant agreement number 223175 (grant number HEALTH-F2-2009-223175) (COGS). Funding for the iCOGS infrastructure came from: the European Community's Seventh Framework Programme under grant agreement number 223175 (HEALTH-F22009-223175) (COGS), Cancer Research UK (C1287/A10118, C1287/A 10710, C12292/A11174, C1281/A12014, C5047/A8384, C5047/A15007, C5047/A10692, C8197/A16565), the National Institutes of Health (CA128978) and Post-Cancer GWAS initiative (1U19 CA148537, 1 U19 CA148065 and 1 U19 CA148112 - the GAME-ON initiative), the Department of Defense (W81XWH-10-1-0341), the Canadian Institutes of Health Research (CIHR) for the CIHR Team in Familial Risks of Breast Cancer, Komen Foundation for the Cure, the Breast Cancer Research Foundation, and the Ovarian Cancer Research Fund. pKARMA was supported by Märit and Hans Rausings Initiative Against Breast Cancer. MCBCS was supported by the NIH grants CA128978, CA116167, CA176785 an NIH Specialized Program of Research Excellence (SPORE) in Breast Cancer (CA116201), and the Breast Cancer Research Foundation and a generous gift from the David F. and Margaret T. Grohne Family Foundation. SEARCH is funded by a programme grant from Cancer Research UK (C490/A10124) and supported by the UK National Institute for Health Research Biomedical Research Centre at the University of Cambridge. HEBCS was financially supported by the Helsinki University Central Hospital Research Fund, Academy of Finland (266528), the Finnish Cancer Society, The Nordic Cancer Union and the Sigrid Juselius Foundation. The MARIE study was supported by the Deutsche Krebshilfe e.V. (70-2892-BR I, 106332, 108253, 108419), the Hamburg Cancer Society, the German Cancer Research Centre (DKFZ) and the Federal Ministry of Education and Research (BMBF) Germany (01KH0402). The CECILE study was funded by Fondation de France, Institut National du Cancer (INCa), Ligue Nationale contre le Cancer, Ligue contre le Cancer Grand Ouest, Agence Nationale de Sécurité Sanitaire (ANSES), Agence Nationale de la Recherche (ANR). BBCS is funded by Cancer Research UK and Breakthrough Breast Cancer and acknowledges NHS funding to the NIHR Biomedical Research Centre, and the National Cancer Research Network (NCRN). kConFab is supported by a grant from the National Breast Cancer Foundation, and previously by the National Health and Medical Research Council (NHMRC), the Queensland Cancer Fund, the Cancer Councils of New South Wales, Victoria, Tasmania and South Australia, and the Cancer Foundation of Western Australia. Financial support for the AOCS was provided by the United States Army Medical Research and Materiel Command (DAMD17-01-1-0729), Cancer Council Victoria, Queensland Cancer Fund, Cancer Council New South Wales, Cancer Council South Australia, The Cancer Foundation of Western Australia, Cancer Council Tasmania and the National Health and Medical Research Council of Australia (NHMRC; 400413, 400281, 199600). GCT and PW are supported by the NHMRC. RB was a Cancer Institute NSW Clinical Research Fellow. TNBCC (RPCI) was supported by a Specialized Program of Research Excellence (SPORE) in Breast Cancer (CA116201), a grant from the Breast Cancer Research Foundation, a generous gift from the David F. and Margaret T. Grohne Family Foundation, and a Cancer Center Support Grant Shared Resource (P30 CA016056-32) for RPCI. The CGPS was supported by the Chief Physician Johan Boserup and Lise Boserup Fund, the Danish Medical Research Council and Herlev Hospital. The ABCS study was supported by the Dutch Cancer Society (grants NKI 20073839; 2009 4363); BBMRI-NL, which is a Research Infrastructure financed by the Dutch government (NWO 184.021.007); and the Dutch National Genomics Initiative. The SBCS was supported by Yorkshire Cancer Research S295, S299, S305PA and Sheffield Experimental Cancer Medicine Centre. The UKBGS is funded by Breast Cancer Now and the Institute of Cancer Research (ICR), London. ICR acknowledges NHS funding to the NIHR Biomedical Research Centre. LMBC is supported by the Stichting tegen Kanker (232-2008 and 1962010). Diether Lambrechts is supported by the FWO and the KULPFV/10/016SymBioSysll. RBCS was funded by the Dutch Cancer Society (DDHK 2004-3124, DDHK 2009-4318). The BSUCH study was supported by the Dietmar-Hopp Foundation, the Helmholtz Society and the German Cancer Research Center (DKFZ). MBCSG is supported by grants from the Italian Association for Cancer Research (AIRC) and by funds from the Italian citizens who allocated the 5/1000 
share of their tax payment in support of the Fondazione IRCCS Istituto Nazionale Tumori, according to Italian laws (INT-Institutional strategic projects " $5 \times 1000$ "). KBCP was financially supported by the special Government Funding (EVO) of Kuopio University Hospital grants, Cancer Fund of North Savo, the Finnish Cancer Organizations, and by the strategic funding of the University of Eastern Finland. MEC was support by NIH grants CA63464, CA54281, CA098758 and CA132839. The ORIGO study was supported by the Dutch Cancer Society (RUL 1997-1505) and the Biobanking and Biomolecular Resources Research Infrastructure (BBMRI-NL CP16) The CNIO-BCS was supported by the Instituto de Salud Carlos III, the Red Temática de Investigación Cooperativa en Cáncer and grants from the Asociación Española Contra el Cáncer and the Fondo de Investigación Sanitario (PI11/00923 and PI12/00070). The Ontario Familial Breast Cancer Registry (OFBCR) was supported by grant UM1 CA164920 from the National Cancer Institute (USA). The content of this manuscript does not necessarily reflect the views or policies of the National Cancer Institute or any of the collaborating centers in the Breast Cancer Family Registry (BCFR), nor does mention of trade names, commercial products, or organizations imply endorsement by the USA Government or the BCFR. The SZBCS was supported by Grant PBZ_KBN_122/P05/2004 The work of the BBCC was partly funded by ELAN-Fond of the University Hospital of Erlangen. OBCS was supported by the Academy of Finland (grant number 250083, 122715 and Center of Excellence grant number 284605), the Finnish Cancer Foundation, the Sigrid Juselius Foundation, the University of Oulu, the University of Oulu Support Foundation and the special Governmental EVO funds for Oulu University Hospital-based research activities. The ESTHER study was supported by a grant from the Baden Württemberg Ministry of Science, Research and Arts. Additional cases were recruited in the context of the VERDI study, which was supported by a grant from the German Cancer Aid (Deutsche Krebshilfe). The GC-HBOC (German Consortium of Hereditary Breast and Ovarian Cancer) is supported by the German Cancer Aid (grant no 110837, coordinator: Rita K. Schmutzler). SKKDKFZS is supported by the DKFZ. HMBCS was supported by a grant from the Friends of Hannover Medical School and by the Rudolf Bartling Foundation. The Australian Breast Cancer Family Study (ABCFS) was supported by grant UM1 CA164920 from the National Cancer Institute (USA). The content of this manuscript does not necessarily reflect the views or policies of the National Cancer Institute or any of the collaborating centers in the Breast Cancer Family Registry (BCFR), nor does mention of trade names, commercial products, or organizations imply endorsement by the USA Government or the BCFR. The ABCFS is also supported by the National Health and Medical Research Council of Australia, the New South Wales Cancer Council, the Victorian Health Promotion Foundation (Australia) and the Victorian Breast Cancer Research Consortium. JLH is a National Health and Medical Research Council (NHMRC) Australia Fellow and a Victorian Breast Cancer Research Consortium Group Leader. MCS is a NHMRC Senior Research Fellow and a Victorian Breast Cancer Research Consortium Group Leader. GENICA was funded by the Federal Ministry of Education and Research (BMBF) Germany grants 01KW9975/5, 01 KW9976/8, 01KW9977/0 and 01KW0114, the Robert Bosch Foundation, Stuttgart, Deutsches Krebsforschungszentrum (DKFZ), Heidelberg, the Institute for Prevention and Occupational Medicine of the German Social Accident Insurance, Institute of the Ruhr University Bochum (IPA), Bochum, and the Department of Internal Medicine, Evangelische Kliniken Bonn gGmbH, Johanniter Krankenhaus, Bonn, Germany. Financial support for KARBAC was provided through the regional agreement on medical training and clinical research (ALF) between Stockholm County Council and Karolinska Institutet, the Swedish Cancer Society, The Gustav V Jubilee foundation and and Bert von Kantzows foundation. MCCS cohort recruitment was funded by VicHealth and Cancer Council Victoria. The MCCS was further supported by Australian NHMRC grants 209057, 251553 and 504711 and by infrastructure provided by Cancer Council Victoria. Cases and their vital status were ascertained through the Victorian Cancer Registry (VCR). The work of MTLGEBCS was supported by the Quebec Breast Cancer Foundation, the Canadian Institutes of Health Research for the CIHR Team in Familial Risks of Breast Cancer programme - grant number CRN-87521 and the Ministry of Economic Development, Innovation and Export Trade - grant number PSR-SIIRI701. The NBCS has received funding from the K.G. Jebsen Centre for Breast Cancer Research; the Research Council of Norway grant 193387N50 (to A-L Børresen-Dale and V.N. Kristensen) and grant 193387/H10 (to A-L Børresen-Dale and V.N. Kristensen), South Eastern Norway Health Authority (grant 39346 to A-L Børresen-Dale) and the Norwegian Cancer Society (to A-L Børresen-Dale and

V.N. Kristensen). PBCS was funded by Intramural Research Funds of the National Cancer Institute, Department of Health and Human Services, USA. The SASBAC study was supported by funding from the Agency for Science, Technology and

ABCS

$\mathrm{BBCC}$

BBCS

BIGGS

BSUCH

CECILE

CGPS

CNIO-BCS

ESTHER

GC-HBOC

HEBCS

HMBCS

ICICLE

KBCP

kConFab/AOCS

MBCSG

MCBCS

MEC

OBCS

OFBCR

ORIGO

pKARMA

RBCS

SBCS

SEARCH

SZBCS

UKBGS
Leiden University Medical Center (LUMC) Commissie Medische Ethiek and Protocol Toetsingscommissie van het Nederlands Kanker Instituut/Antoni van Leeuwenhoek Ziekenhuis

Friedrich-Alexander-Universitat Erlangen-Nurnberg Medizinische Fakultat Ethik-Commission

South East Multi-Centre Research Ethics Committee

Galway University College Hospital Clinical Research Ethical Committee

Medizinische Fakultat Heidelberg Ethikkommission

Comite Consultatif de Protection des Personnes dans la Recherche Biomedicale de Bicetre

Kobenhavns Amt den Videnskabsetiske Komite

Hospital Universitario La Paz Comite Etico de Investigacion Clinica

Ruprecht-Karls-Universitat Medizinische Fakultat Heidelberg Ethikkommission

Ethik-Kommission der Medizinischen Fakultat der Universitat zu Koln

Helsingin ja uudenmaan sairaanhoitopiiri (Helsinki University Central Hospital Ethics Committee)

Medizinische Hochschule Hannover Ethik-Kommission

Southampton and South West Hampshire Research Ethics Committee A (MREC 08/H0502/4)

Pohjois-Savon Sairraanhoitopiirin Kuntayhtyma Tutkimuseettinen Toimikunta

kConFab: The Queenland Institute of Medical Research Human Research Ethics Committee (QIMR-HREC)

AOCS: Peter MacCallum Cancer Centre Ethics Committee

Ruprecht-Karls-Universitat Medizinische Fakultat Heidelberg Ethikkommission

Comitato Etico Indipendente della Fondazione IRCCS "Istituto Nazionale dei Tumori"

Mayo Clinic IRB

University of Southern California Health Sciences Campus IRB

Ethical Committee of the Medical Faculty of University of Oulu and Northern Ostrobothnia Hospital District Ethical Committee

Mount Sinai Hospital Research Ethics Board

Medical Ethical Committee and Board of Directors of the Leiden University Medical Center (LUMC)

Regionala Etikprovningsnamnden i Stockholm (Regional Ethical Review Board in Stockholm)

Medische Ethische Toetsings Commissie Erasmus Medisch Centrum

South Sheffield Research Ethics Committee

Multi Centre Research Ethics Committee (MREC)

Komisji Bioetycznej Pomorskiej Akademii Medycznej

South East Multi-Centre Research Ethics Committee 
Research of Singapore (A*STAR), the US National Institute of Health (NIH) and the Susan G. Komen Breast Cancer Foundation. Ethical approval bodies for BCAC studies are listed below.

\section{Funding}

ICICLE genotyping was funded by the Breast Cancer Now

(http://breastcancernow.org/), and sample and data collection.

\section{Author details}

${ }^{1}$ Research Oncology, Guy's Hospital, King's College London, London, UK. ${ }^{2}$ Medical and Molecular Genetics, Guy's Hospital, King's College London, London, UK. ${ }^{3}$ Division of Genetics and Epidemiology, The Institute of Cancer Research, London, UK. ${ }^{4}$ Centre for Molecular Oncology, Barts Cancer Institute, Queen Mary University of London, London, UK. ${ }^{5}$ Biomedical Research Centre, King's College London, Guy's Hospital, London, UK. ${ }^{6}$ The Breast Cancer Now Toby Robins Research Centre, The Institute of Cancer Research, London, UK. ${ }^{7}$ Sheffield Cancer Research, Department of Oncology, University of Sheffield, Sheffield, UK. ${ }^{8}$ Academic Unit of Pathology, Department of Neuroscience, University of Sheffield, Sheffield, UK. ${ }^{9}$ Department of Non-Communicable Disease Epidemiology, London School of Hygiene and Tropical Medicine, London, UK. ${ }^{10}$ Division of Breast Cancer Research, The Institute of Cancer Research, London, UK. ${ }^{11}$ Centre for Cancer Genetic Epidemiology, Department of Public Health and Primary Care, University of Cambridge, Cambridge, UK. ${ }^{12}$ Human Cancer Genetics Program, Spanish National Cancer Research Centre, Madrid, Spain. ${ }^{13}$ Centro de Investigación en Red de Enfermedades Raras, Valencia, Spain. ${ }^{14}$ Centre d'innovation Génome Québec et Université McGill, Montréal, Canada. ${ }^{15}$ Department of Medical Epidemiology and Biostatistics, Karolinska Institutet, Stockholm, Sweden. ${ }^{16}$ Division of Cancer Epidemiology and Genetics, National Cancer Institute, Rockville, MD, USA. ${ }^{17}$ Department of Genetics, Institute for Cancer Research, Oslo University Hospital Radiumhospitalet, Oslo, Norway. ${ }^{18} \mathrm{~K}$.G. Jebsen Center for Breast Cancer Research, Institute of Clinical Medicine, Faculty of Medicine, University of Oslo, Oslo, Norway. ${ }^{19}$ Department of Clinical Molecular Biology, Oslo University Hospital, University of Oslo, Oslo, Norway. ${ }^{20}$ Genomics Center, Centre Hospitalier Universitaire de Québec Research Center, Laval University, Québec City, Canada. ${ }^{21}$ Cancer Epidemiology Centre, Cancer Council Victoria, Melbourne, Australia. ${ }^{22}$ Centre for Epidemiology and Biostatistics, Melbourne School of Population and Global health, The University of Melbourne, Melbourne, VIC, Australia. ${ }^{23}$ Department of Oncology - Pathology, Karolinska Institutet, Stockholm, Sweden. ${ }^{24}$ Department of Molecular Medicine and Surgery, Karolinska Institutet, Stockholm, Sweden. ${ }^{25}$ Institute for Prevention and Occupational Medicine of the German Social Accident Insurance, Institute of the Ruhr University Bochum, Bochum, Germany. ${ }^{26}$ Dr. Margarete Fischer-Bosch-Institute of Clinical Pharmacology, Stuttgart, Germany. ${ }^{27}$ University of Tübingen, Tübingen, Germany. ${ }^{28}$ German Cancer Consortium (DKTK), German Cancer Research Center (DKFZ), Heidelberg, Germany. ${ }^{29}$ Department of Pathology, The University of Melbourne, Melbourne, Australia. ${ }^{30}$ Gynaecology Research Unit, Hannover Medical School, Hannover, Germany. ${ }^{31}$ Department of Radiation Oncology, Hannover Medical School, Hannover, Germany. ${ }^{32}$ Molecular Genetics of Breast Cancer, German Cancer Research Center (DKFZ), Heidelberg, Germany. ${ }^{33}$ Center for Familial Breast and Ovarian Cancer, Medical Faculty, University of Cologne and University Hospital Cologne, Cologne, Germany. ${ }^{34} \mathrm{Center}$ for Integrated Oncology (CIO), Medical Faculty, University of Cologne and University Hospital Cologne, Cologne, Germany. ${ }^{35}$ Center for Molecular Medicine Cologne (CMMC), Medical Faculty, University of Cologne and University Hospital Cologne, Cologne, Germany. ${ }^{36}$ Division of Gynaecology and Obstetrics, Technische Universität München, Munich, Germany. ${ }^{37}$ Division of Clinical Epidemiology and Aging Research, German Cancer Research Center (DKFZ), Heidelberg, Germany. ${ }^{38}$ Division of Preventive Oncology, German Cancer Research Center (DKFZ), Heidelberg, Germany. ${ }^{39}$ Laboratory of Cancer Genetics and Tumor Biology, Cancer and Translational Medicine Research Unit, Biocenter Oulu, University of Oulu, Oulu, Finland. ${ }^{40}$ Laboratory of Cancer Genetics and Tumor Biology, Northern Finland Laboratory Centre NordLab, Oulu, Finland. ${ }^{41}$ Department of Gynaecology and Obstetrics, University Hospital Erlangen, Friedrich-Alexander University Erlangen-Nuremberg, Comprehensive Cancer Center Erlangen-EMN, Erlangen, Germany. ${ }^{42}$ David Geffen School of Medicine, Department of Medicine Division of Hematology and Oncology, University of California at Los Angeles, Los Angeles, CA, USA. ${ }^{43}$ Department of Genetics and Pathology, Pomeranian Medical University, Szczecin, Poland. ${ }^{44}$ Department of Laboratory Medicine and Pathobiology, University of
Toronto, Toronto, Canada. ${ }^{45}$ Laboratory Medicine Program, University Health Network, Toronto, Canada. ${ }^{46}$ Lunenfeld-Tanenbaum Research Institute of Mount Sinai Hospital, Toronto, Canada. ${ }^{47}$ Department of Molecular Genetics, University of Toronto, Toronto, Canada. ${ }^{48}$ Department of Surgery, Leiden University Medical Center, Leiden, The Netherlands. ${ }^{49}$ Department of Pathology, Leiden University Medical Center, Leiden, The Netherlands. ${ }^{50}$ Department of Human Genetics, Leiden University Medical Center, Leiden, The Netherlands. ${ }^{51}$ University of Hawaii Cancer Center, Honolulu, HI, USA. ${ }^{52}$ Department of Preventive Medicine, Keck School of Medicine, University of Southern California, Los Angeles, CA, USA. ${ }^{53}$ Imaging Center, Department of Clinical Pathology, Kuopio University Hospital, Kuopio, Finland. ${ }^{54}$ Institute of Clinical Medicine, Pathology and Forensic Medicine, University of Eastern Finland, Kuopio, Finland. ${ }^{55}$ Cancer Center of Eastern Finland, University of Eastern Finland, Kuopio, Finland. ${ }^{56}$ Unit of Molecular Bases of Genetic Risk and Genetic Testing, Department of Preventive and Predictive Medicine, Fondazione IRCCS (Istituto Di Ricovero e Cura a Carattere Scientifico) Istituto Nazionale dei Tumori (INT), Milan, Italy. ${ }^{57}$ IFOM, Fondazione Istituto FIRC (Italian Foundation of Cancer Research) di Oncologia Molecolare, Milan, Italy. ${ }^{58}$ National Center for Tumor Diseases, University of Heidelberg, Heidelberg, Germany. ${ }^{59}$ Department of Obstetrics and Gynecology, University of Heidelberg, Heidelberg, Germany. ${ }^{60}$ Molecular Epidemiology Group, German Cancer Research Center (DKFZ), Heidelberg, Germany. ${ }^{61}$ Department of Pathology, Erasmus University Medical Center, Rotterdam, The Netherlands. ${ }^{62}$ Department of Medical Oncology, Family Cancer Clinic, Erasmus MC Cancer Institute, Rotterdam, The Netherlands. ${ }^{63}$ School of Medicine, National University of Ireland, Galway, Ireland. ${ }^{64}$ Vesalius Research Center, VIB, Leuven, Belgium. ${ }^{65}$ Laboratory for Translational Genetics, Department of Oncology, University of Leuven, Leuven, Belgium. ${ }^{66}$ University Hospital Gashuisberg, Leuven, Belgium. ${ }^{67}$ Netherlands Cancer Institute, Antoni van Leeuwenhoek hospital, Amsterdam, The Netherlands. ${ }^{68}$ Department of Breast Surgery, Herlev Hospital, Copenhagen University Hospital, Herlev, Denmark. ${ }^{69}$ Copenhagen General Population Study, Herlev Hospital, Copenhagen University Hospital, Herlev, Denmark. ${ }^{70}$ Department of Clinical Biochemistry, Herlev Hospital, Copenhagen University Hospital, Herlev, Denmark. ${ }^{71}$ Faculty of Health and Medical Sciences, University of Copenhagen, Copenhagen, Denmark. ${ }^{72}$ Department of Cancer Prevention and Control, Roswell Park Cancer Institute, Buffalo, NY, USA. ${ }^{73}$ Roswell Park Cancer Institute, Buffalo, NY, USA. ${ }^{74}$ Department of Genetics, QIMR Berghofer Medical Research Institute, Brisbane, Australia. ${ }^{75}$ Environmental Epidemiology of Cancer, Center for Research in Epidemiology and Population Health, INSERM, Villejuif, France. ${ }^{76}$ University Paris-Sud, Villejuif, France. ${ }^{77}$ Division of Cancer Epidemiology, German Cancer Research Center (DKFZ), Heidelberg, Germany. ${ }^{78}$ Department of Obstetrics and Gynecology, Helsinki University Hospital, University of Helsinki, Helsinki, Finland. ${ }^{79}$ Department of Oncology, Helsinki University Hospital, University of Helsinki, Helsinki, Finland. ${ }^{80}$ Department of Health Sciences Research, Mayo Clinic, Rochester, MN, USA. ${ }^{81}$ Department of Laboratory Medicine and Pathology, Mayo Clinic, Rochester, MN, USA. ${ }^{82}$ Centre for Cancer Genetic Epidemiology, Department of Oncology, University of Cambridge, Cambridge, UK. ${ }^{83}$ Wellcome Trust Centre for Human Genetics and Oxford NIHR Biomedical Research Centre, University of Oxford, Oxford, UK.

Received: 1 September 2015 Accepted: 6 January 2016

Published online: 17 February 2016

\section{References}

1. Li Cl, Daling JR, Malone KE. Age-specific incidence rates of in situ breast carcinomas by histologic type, 1980 to. Cancer Epidemiol Biomarkers Prev. 2001;14(4):1008-11.

2. Leonard GD, Swain SM. Ductal carcinoma in situ, complexities and challenges. J Natl Cancer Inst. 2004;96(12):906-20.

3. Wong H, Lau S, Yau T, Cheung P, Epstein RJ. Presence of an in situ component is associated with reduced biological aggressiveness of size-matched invasive breast cancer. Brit J Cancer. 2010;102(9):1391-6.

4. Ruszczyk M, Zirpoli G, Kumar S, Bandera EV, Bovbjerg DH, Jandorf L, et al. Breast cancer risk factor associations differ for pure versus invasive carcinoma with an in situ component in case-control and case-case analyses. Cancer Causes Control. http://dx.doi.org/10.1007/ s10552-015-0696-z. 
5. Li Cl, Malone KE, Saltzman BS, Daling JR. Risk of invasive breast carcinoma among women diagnosed with ductal carcinoma in situ and lobular carcinoma in situ, 1988-2001. Cancer. 2006;106(10):2104-12.

6. Collins LC, Tamimi RM, Baer HJ, Connolly JL, Colditz GA, Schnitt SJ. Outcome of patients with ductal carcinoma in situ untreated after diagnostic biopsy: results from the Nurses' Health Study. Cancer. 2005 103(9):1778-84.

7. Wapnir IL, Dignam JJ, Fisher B, Mamounas EP, Anderson SJ, Julian TB, et al. Long-term outcomes of invasive ipsilateral breast tumor recurrences after lumpectomy in NSABP B-17 and B-24 randomized clinical trials for DCIS. J Natl Cancer Inst. 2011;103(6):478-88.

8. Pinder SE. Ductal carcinoma in situ (DCIS): pathological features, differential diagnosis, prognostic factors and specimen evaluation. Mod Pathol. 2010;23 Suppl 2:S8-13.

9. Aguiar FN, Mendes HN, Bacchi CE, Carvalho FM. Comparison of nuclear grade and immunohistochemical features in situ and invasive components of ductal carcinoma of breast. Rev Bras Ginecol Obstet. 2013;35(3):97-102.

10. Leong AS, Sormunen RT, Vinyuvat S, Hamdani RW, Suthipintawong C. Biologic markers in ductal carcinoma in situ and concurrent infiltrating carcinoma. A comparison of eight contemporary grading systems. Am J Clin Pathol. 2001:115(5):709-18.

11. Claus EB, Stowe M, Carter D. Breast carcinoma in situ: risk factors and screening patterns. J Natl Cancer Inst. 2001;93(23):1811-7.

12. Reeves GK, Pirie K, Green J, Bull D, Beral V, Million Women Study C. Comparison of the effects of genetic and environmental risk factors on in situ and invasive ductal breast cancer. Int J Cancer. 2012;131(4):930-7.

13. Claus EB, Stowe M, Carter D. Family history of breast and ovarian cancer and the risk of breast carcinoma in situ. Breast Cancer Res Treat. 2003;78(1):7-15.

14. Kerlikowske K, Barclay J, Grady D, Sickles EA, Ernster V. Comparison of risk factors for ductal carcinoma in situ and invasive breast cancer. J Natl Cancer Inst. 1997;89(1):76-82.

15. Claus EB, Petruzella S, Matloff E, Carter D. Prevalence of BRCA1 and BRCA2 mutations in women diagnosed with ductal carcinoma in situ. JAMA. 2005;293(8):964-9.

16. Sawyer E, Roylance R, Petridis C, Brook MN, Nowinski S, Papouli E, et al. Genetic predisposition to in situ and invasive lobular carcinoma of the breast. PLoS Genet. 2014;10(4):e1004285.

17. Purrington KS, Slager S, Eccles D, Yannoukakos D, Fasching PA, Miron P, et al. Genome-wide association study identifies 25 known breast cancer susceptibility loci as risk factors for triple-negative breast cancer. Carcinogenesis. 2014;35(5):1012-9.

18. Garcia-Closas M, Couch FJ, Lindstrom S, Michailidou K, Schmidt MK, Brook $M N$, et al. Genome-wide association studies identify four ER negativespecific breast cancer risk loci. Nat Genet. 2013;45(4):392-8. 398e391-392.

19. Michailidou K, Hall P, Gonzalez-Neira A, Ghoussaini M, Dennis J, Milne RL et al. Large-scale genotyping identifies 41 new loci associated with breast cancer risk. Nat Genet. 2013;45(4):353-61. 361e351-352.

20. Willer CJ, Li Y, Abecasis GR. METAL: fast and efficient meta-analysis of genome wide association scans. Bioinformatics. 2010;26(17):2190-1.

21. Siddiq A, Couch FJ, Chen GK, Lindstrom S, Eccles D, Millikan RC, et al. A meta-analysis of genome-wide association studies of breast cancer identifies two novel susceptibility loci at $6 q 14$ and 20q11. Hum Mol Genet. 2012;21(24):5373-84.

22. Lester SC, Bose S, Chen YY, Connolly JL, de Baca ME, Fitzgibbons PL, et al. Protocol for the examination of specimens from patients with ductal carcinoma in situ of the breast. Arch Pathol Lab Med. 2009;133(1):15-25.

23. Milne RL, Goode EL, Garca-Closas M, Couch FJ, Severi G, Hein R, et al. Confirmation of 5 p12 as a susceptibility locus for progesterone-receptorpositive, lower grade breast cancer. Cancer Epidemiol Biomarkers Prev. 2011;20(10):2222-31.

24. Easton DF, Pooley KA, Dunning AM, Pharoah PD, Thompson D, Ballinger DG, et al. Genome-wide association study identifies novel breast cancer susceptibility loci. Nature. 2007:447(7148):1087-93.

25. Campa D, Barrdahl M, Gaudet MM, Black A, Chanock SJ, Diver WR, et al. Genetic risk variants associated with in situ breast cancer. Breast Cancer Res. 2015;17:82.

26. Milne RL, Burwinkel B, Michailidou K, Arias-Perez JI, Zamora MP, MenendezRodriguez $\mathrm{P}$, et al. Common non-synonymous SNPs associated with breast cancer susceptibility: findings from the Breast Cancer Association Consortium. Hum Mol Genet. 2014;23(22):6096-111.
27. Thomas J, Hanby A, Pinder S, Ellis I, Macartney J, Clements K, et al. Implications of inconsistent measurement of ER status in non-invasive breast cancer: a study of 1,684 cases from the Sloane Project. Breast J. 2008;14(1):33-8.

28. Garcia-Closas M, Hall P, Nevanlinna H, Pooley K, Morrison J, Richesson DA, et al. Heterogeneity of breast cancer associations with five susceptibility loci by clinical and pathological characteristics. PLoS Genet. 2008;4(4):e1000054.

29. Purrington KS, Slettedahl S, Bolla MK, Michailidou K, Czene K, Nevanlinna H, et al. Genetic variation in mitotic regulatory pathway genes is associated with breast tumor grade. Hum Mol Genet. 2014;23(22):6034-46.

30. Lambrechts D, Truong T, Justenhoven C, Humphreys MK, Wang J, Hopper $J$, et al. 11 q13 is a susceptibility locus for hormone receptor positive breast cancer. Hum Mutat. 2012;33(7):1123-32.

31. French JD, Ghoussaini M, Edwards SL, Meyer KB, Michailidou K, Ahmed S, et al. Functional variants at the 11 q13 risk locus for breast cancer regulate Cyclin D1 expression through long-range enhancers. Am J Hum Genet. 2013;92(4):489-503.

32. Arvold ND, Punglia RS, Hughes ME, Jiang W, Edge SB, Javid SH, et al. Pathologic characteristics of second breast cancers after breast conservation for ductal carcinoma in situ. Cancer. 2012;118(24):6022-30.

\section{Submit your next manuscript to BioMed Central and we will help you at every step:}

- We accept pre-submission inquiries

- Our selector tool helps you to find the most relevant journal

- We provide round the clock customer support

- Convenient online submission

- Thorough peer review

- Inclusion in PubMed and all major indexing services

- Maximum visibility for your research

Submit your manuscript at www.biomedcentral.com/submit
) Biomed Central 